\title{
MODULATION OF ELECTRON WAVES BY MEANS OF A LASER
}

\author{
Ph.D. Thesis Submitted to lowa State University, \\ November, 1972
}

\author{
E. J. Stanek II
}

\author{
Ames Laboratory, USAEC \\ lowa State University \\ Ames, lowa 50010
}

Date of Manuscript: November, 1972

PREPARED FOR THE U. S. ATOMIC ENERGY COMMISSION DIVISION OF RESEARCH UNDER CONTRACT NO. W-7405-eng-82 


\section{DISCLAIMER}

This report was prepared as an account of work sponsored by an agency of the United States Government. Neither the United States Government nor any agency Thereof, nor any of their employees, makes any warranty, express or implied, or assumes any legal liability or responsibility for the accuracy, completeness, or usefulness of any information, apparatus, product, or process disclosed, or represents that its use would not infringe privately owned rights. Reference herein to any specific commercial product, process, or service by trade name, trademark, manufacturer, or otherwise does not necessarily constitute or imply its endorsement, recommendation, or favoring by the United States Government or any agency thereof. The views and opinions of authors expressed herein do not necessarily state or reflect those of the United States Government or any agency thereof. 


\section{DISCLAIMER}

Portions of this document may be illegible in electronic image products. Images are produced from the best available original document. 


This report was prepared as an account of work
sponsored by the United States Government. Neither
the United States nor the United States Atomic
Energy Commission, nor any of their employees, nor
any of their contractors, subcontractors, or their
employees, makes any warranty, express or implied,
or assumes any legal liability or responsibility for the
accuracy, completeness or usefulness of any
information, apparatus, product or process disclosed,
or represents that its use would not infringe privately
owned rights.

Available from: National Technical Information Service Department A

Springfield, VA 22151

Price: Microfiche $\$ 0.95$ 

Modulation of electron waves by means of a laser
by
Edward Joseph Stanek II

A Dissertation Submitted to the

Graduate Faculty in Partial Fulfillment of

The Requirements for the Degree of

DOCTOR OF PHILOSOPHY

Department: Physics

Major: Physics

Approved:

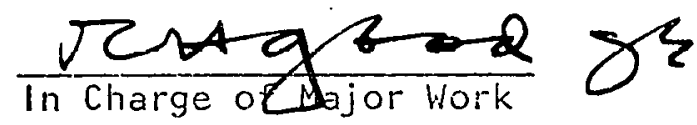

$\frac{\text { POMarnef }}{\text { For the Hajor Department }}$

Charlite Exodemck

For the Graduate Coilege

'lowa State University

Ames, lowa 


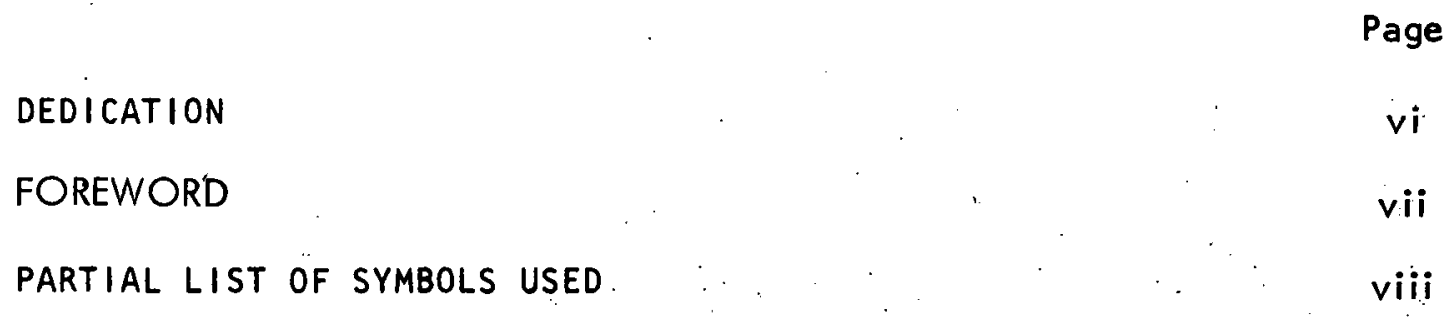

I. INTRODUCTION I

A. The Schwarz-Hora Effect 1

B. Speculative Explanations 4

C. Need for More Experimental Data 5

D. Spread of Experimental Interest 5

il. THE FIRST EXPERIMENT AT I.S.U. 8

A. Apparatus and Procedure 8

B. Analysis and Some New Revelations 13

III. THE SECOND EXPERIMENT AT I.S.U. 17.

A. Apparatus 17

B. Film Samples 26

C. A Final Modification 33

D. Procedure 34

E. An Analysis of Results $\quad 37$

IV. DISCUSSION 40

V. SYNOPSIS OF THEORETICAL PROGRESS AND MOTIVATION
FOR CONTINUED WORK

A. Theoretical Advancements $\quad 47$

B. Coherence Criterion 50 
VI. RELATIVISTIC THEORY OF THE SCHWARZ-HORA TYPE MODULATION MECHANISM

A. The Laser Potential and General Formulas

B. The Undeflected Beam 55

C. The Diffracted Beams 61

$\begin{array}{ll}\text { VII. FINAL REMARKS } & 68\end{array}$

VIII. REFERENCES CITED . 69

IX. ACKNOWLEDGMENT

X. APPENDIX I $\quad 72$

XI. APPENDIX 11 


\section{DEDICATION}

This manuscript is dedicated to my parents, Mr. and Mrs. Edward J. Stanek, for their guidance, support, and concern--and to my wi fe, Jackle, for making all-my troubles surmountable. 


\section{FOREWORD}

This dissertation represents a part of the culmination of two years of research in both experimental and theoretical physics. The initial. enthusiasm for the work on this problem was provided by Professor R. $H$. Good, Jr. Experimental aspects were conducted by the author in collaboration with Professor D. W. Lynch of the lowa State University physics department, Or. G. R. Hadley now at Sandia Corporation, Albuquerque, New Mexico, and Dr. Elmer Rosauer of the ceramics engineering department at lowa State. Parts of the experimental material contained herein were published jointly by the author, Dr. Hadley, Dr. Lynch, and Dr. Rosauer (1). Theoretical aspects were collaborated by the author, Dr. Hadley, and Dr. Good and parts published jointly by the group (2). Another manuscript containing parts of this work was written jointly by the group and has been submitted for publication (3). The author has been unrestrained in expressing his opinion on the following pages. 
viii

PARTIAL LIST OF SYMBOLS USED

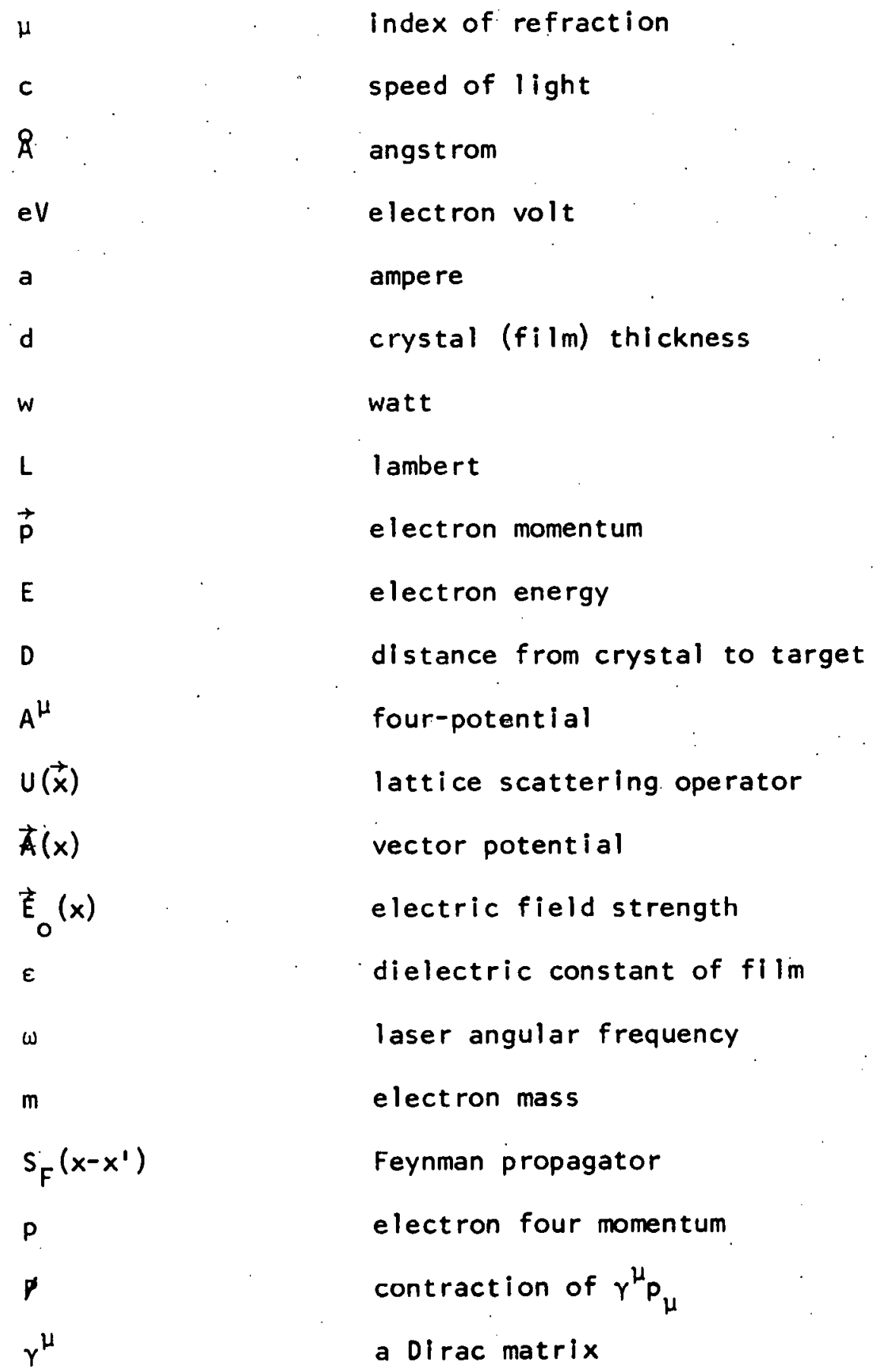


I. INTRODUCTION

A. The Schwarz-Hora Effect

In December of 1969 a paper entitled Modulation of an Electron Wave by a Light Wave" was published in Applied Physics Letters (4). The authors were Helmut Schwarz of the Rensselaer Polytechnic Institute and Heinrich Hora visiting at Rensselaer but since returned to the Institute fur Plasmaphysik, Max Planck Gesel.1schaft, Garching, Germany. They reported an effect in which electron transmission diffraction patterns of thin monocrystal films are made visible on a nonluminescent screen by means of the simultaneous intersection of a laser beam with the electron beam within the crystal (see Figure 1). The report of this phenomenon was to start a cascade of experimental and theoretical research that is continuing at this writing. It was also to open the door to mystery and intrigue - the type of which the American physicist is seldom forced to confront.

According to Reference 4 the following procedure was used to produce the above described effect:

1) Films of $\mathrm{SiO}_{2}$ and $\mathrm{Al}_{2} \mathrm{O}_{3}$ were "epitaxially vacuum-deposited" to thicknesses between 600 and 2,000 $\AA$. These films were floated off their substrates onto special grids.

2) The grid supporting the film was constructed so as not to obstruct a laser beam which could be passed through the crystal and perpendicular to the electron beam.

3) A $50 \mathrm{keV}$ electron beam of about one microampere in a spot of. a few microns diameter was focused on an area of the film which 


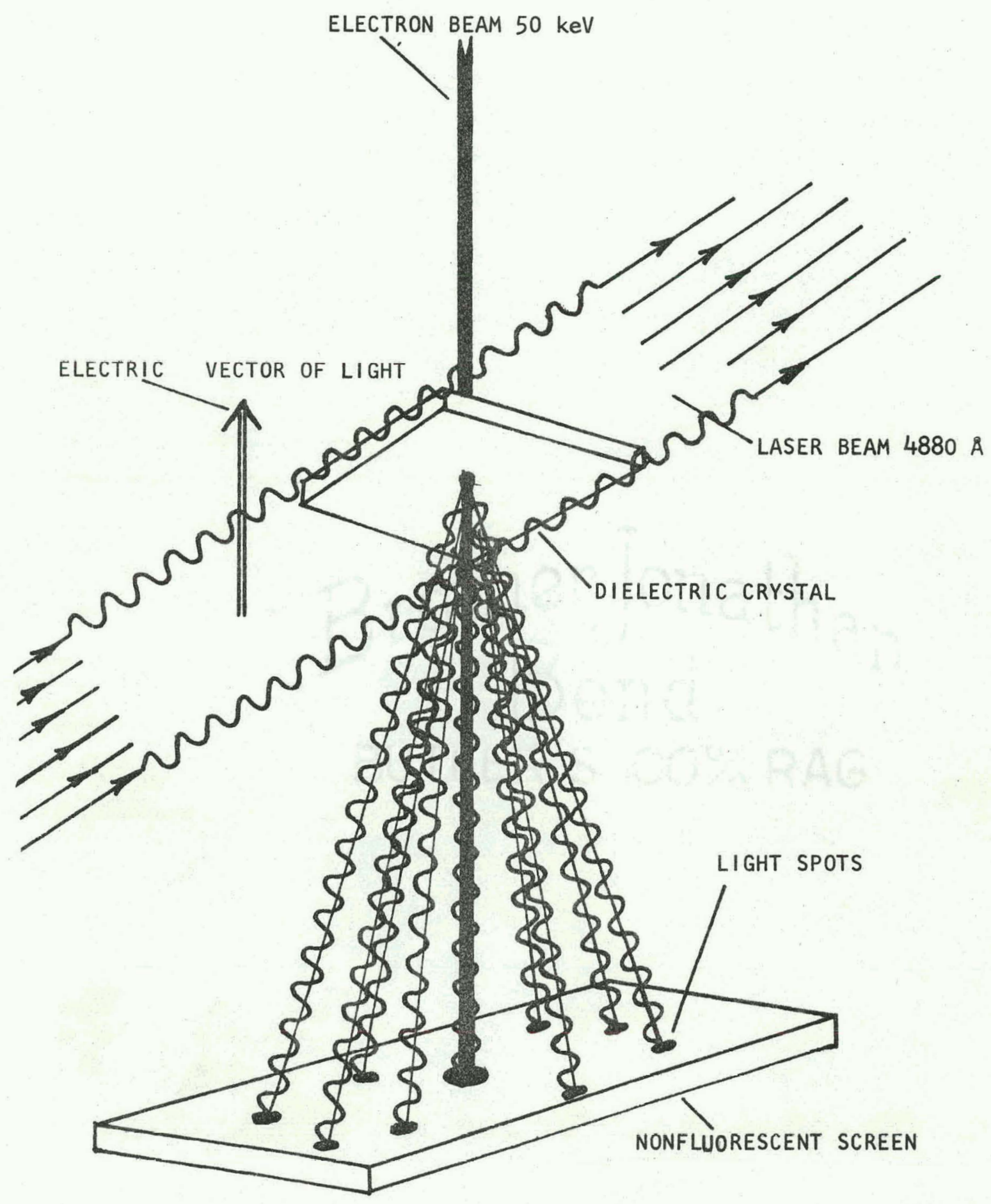

Figure 1. Schematic diagram of the Schwarz-Hora effect 
produced a diffraction pattern indicating a monocrystal structure.

4) The fluorescent screen normally used to observe the diffraction pattern was removed and replaced by a smooth flat sheet of polycrystalline alumina at a distance of $25 \mathrm{~cm}$ from the $\mathrm{film}$ making the diffraction pattern invisible.

5) When the 10 watt laser beam of wavelength $4880 \AA$ was turned on, the diffraction pattern reappeared in the laser color on the nonluminescent sheet.

If the laser beam did not intersect the electron beam within the crystal the effect disappeared (5). The effect also decreased in intensity as the angle between the laser polarization and the electron beam direction increased from 0 to 90 degrees where the effect disappeared altogether. slowing down the electrons and increasing correspondingly their number resulted in a substantial decrease of light intensity on the alumina target whereas light from the fluorescent screen showed no change in over-all intensity. A magnet was used to deflect the electron beam and resulted in displacement of the blue spots.

No further details of the experiment were specified. A picture included in the publication showed the spots on the fluorescent screen to be round and nicely symmetric as expected. However, the spots on the nonluminescent screen had odd shapes and unexplainable areas of brightness and darkness. 


\section{B. Speculative Explanations}

The phenomenon was soon dubbed the Schwarz-Hora effect. Interesting speculations were soon made about its nature. It was apparently evident that the electrons picked up information from the laser beam (specifically its frequency) at the crystal and somehow communicated this information to the screen. Any theory formulated to explain the experiment would have to treat a process at the crystal and another probably very distinct process at the screen. Either or both processes could possibly be either classical or quantum mechanical in nature. Early in 1970 Rubin (6) and 01 iver and Cutler (7) presented a classical modulation mechanism modeled after the klystron. The electrons were to have been physically bunched at the laser frequency. This amplitude modulated d.c. current somehow radiated the laser frequency back when the beam struck its target. A complete analogy with the klystron was impossible because there is no feedback mechanism in the Schwarz-Hora apparatus. It was commonly accepted that the mechanism by which information was picked up by the electrons was modulation. The classical nature of this modulation was soon discarded as erroneous. Using the parameters given in Reference 4 it is easy to see that there were less than 500 electrons per centimeter in the beam. If the electrons were bunched at the laser wavelength of $4880 \AA$ there would only be a small fraction of one electron per bunch - fractions of electrons do not make sense in a classical theory. It appeared as though the modulation must then be of a quantum mechanical nature. 
Early quantum mechanical theories of the modulation were posed by Van Zandt and Meyer (8), Hutson (9), and Salat (10). Varshalovich and D'yakanov (11) as well as Van Zandt (12) assumed a modulated electron wave and posed quantum mechanical explanations of the radiation emitted at the target. No quantitative results were presented except by Hutson who treated the crystal as a wave guide. However, he had to guess at the enhancement of the electromagnetic induction caused by the guided mode.

\section{Need for More Experimental Data}

The repercussions of the Schwarz-Hora effect could open a whole new branch of technology. For decades frequency modulated electromagnetic waves have been used to broadcast radio programs in local areas all over the world. Now for the first time it appeared that particle waves could also be frequency modulated. An aesthetic gleam rose in the eyes of those who ponder particle-wave duality. Hora soon proposed a revolutionary color television mechanism based on his effect (13). Instead of using a multi-electron gun picture tube or different color producing phosphors, a single electron beam would be modulated by a small frequency variable laser or variable plasmon frequency film. The appropriate color would appear at the right place on a nonfluorescent screen.

\section{Spread of Experimental Interest}

Different experimental groups around the country expressed an interest in reproducing the effect. Hewlett-Packard supposedly bargained with Schwarz to obtain a patent on the process. Donald Scarl at Brooklyn 
Polytech set up an experiment with a pulsed laser of lower average power than Schwarz's but much greater power during a given pulse. In order to evade making visual observations, sensitive photomultipliers were used in coincidence with the laser to measure the emitted radiation at the target. At Bell Laboratories in Murray Hill, New Jersey, Loren Pfeiffer and Dennis Rousseau undertook an elaborate attempt to reconstruct Schwarz's apparatus as closely as possible.

At lowa State R. H. Good, Jr., G. R. Hadley, and the author had a theoretical interest in the problem. It was thought that possibly a bremsstrahlung - inverse bremsstrahlung type process excited at the laser frequency when applied to the electron's interactions within the crystal could account for the modulation. Then somehow this different energy beam would combine with the unscattered beam to produce the radiation at the target. The mechanism by which this radiation was produced was not understood and clues were sought in order to get some idea on how to handle it. In a private communication with Schwarz, he revealed that the light at the target was bright enough to see from across the room but he had not measured its intensity or exact frequency. The effect apparently was quite intense and no theoretical ideas with visible intensities in a non resonant target were forthcoming.

It was clear that more data were needed. The aroups at Brooklyn Polytech and Bell Labs had not yet completed the fabrication of their devices. Schwarz had left much undone and was lecturing around the world while his laboratory was being moved. Many questions needed answering. How important was the modulating crystal composition and structure? 
Did it matter what the target was made of? What was the intensity and exact spectrum of the radiation emitted at the target? Was there something magic about the $4880 \&$ laser line? There was an electron microscope at lowa State and at least two powerful lasers. Maybe they could be put together in such a way as to reasonably duplicate Schwarz's apparatus . 


\section{THE FIRST EXPERIMENT AT I.S.U.}

A. Apparatus and Procedure

Dr. David Lynch at $1.5 . U$. expressed an interest in joining the collaboration. Dr. Elmer Rosauer of the Ceramics Engineering Department at lowa State was convinced that the project was worthwhile and consented to give the use of and help to operate his electron microscope. Fred Dechow of the Chemistry Department joined in with his 1 watt argon ion laser and the apparatus in Figure 2 was assembled. 1 The electron beam energy was tried at $40,60,80$, and $100 \mathrm{keV}$ which were the four energy capabilities of the electron microscope. The current was con-

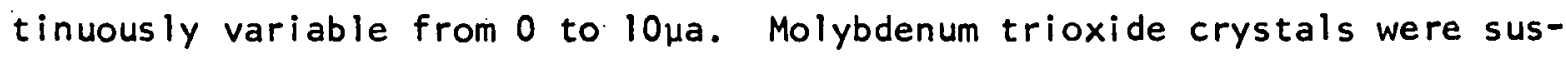
pended on a film coated grid. Schwarz released more information in a preprint $^{2}$ indicating that the effect had also been obtained with crystals of $\mathrm{SrF}_{2}$ as well as the $\mathrm{Al}_{2} \mathrm{O}_{3}$ and $\mathrm{SiO}_{2}$. Since our sample was also a clear transparent crystal there was no reason to suspect that the experiment would be adversely affected. A large enough crystal was selected by using the magnification capability of the Elmiskop I electron microscope. By observation of the magnitifed image of the transmitted electron beam, thin and thick portions of a microscopic crystal could be discerned. Because the thicker portions (or thicker crystals as the case might be)

'A more detailed description of the electron microscope will be presented in the next chapter since a description of additional equipment will be needed there.

2 Eventually published as Reference 5 . 


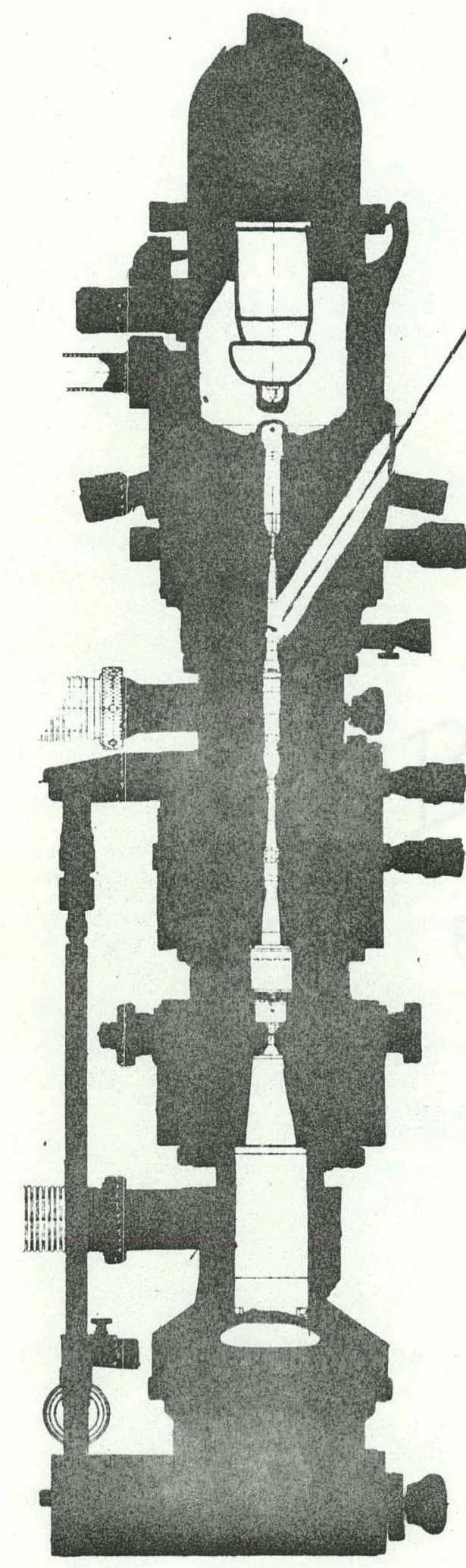

ELECTRON GUN
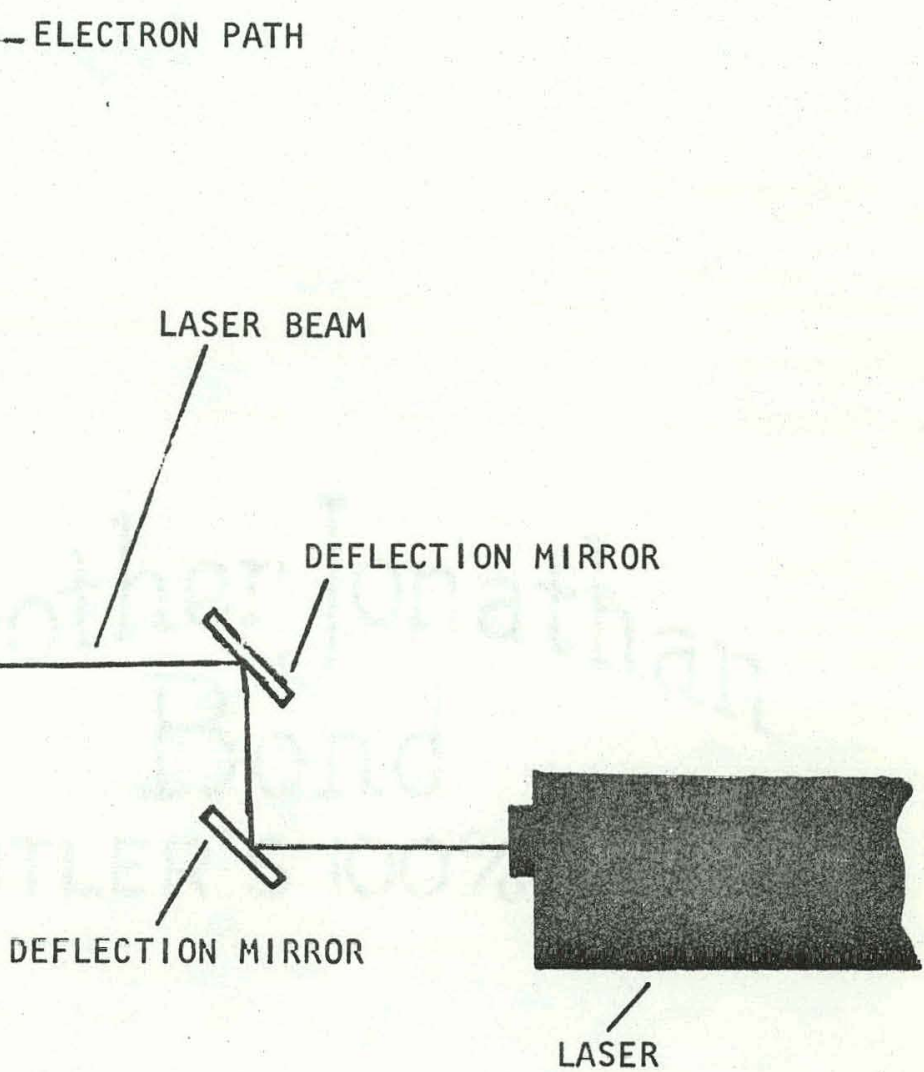

SCREEN VIEWING WINDOW

Figure 2. First experimental apparatus 
absorb a greater percentage of the electron beam than do thinner portions (or thinner crystals) they appear as heavy shadows on the luminescent target. The degree of darkness is proportional to the sample thickness. Light areas which subsequently produced a large number of diffraction spots were judged to be less than a few thousand angstroms in depth. A special quartz' ${ }^{1}$ window was fitted into the side of the sample holder chamber to allow the passage of the laser beam. The beam was about $5 \mathrm{~mm}$ in diameter but reduced to a fine focus by a glass lens before entering the microscope. The focused beam covered about $1 \mathrm{~mm}^{2}$ of the $2.3 \mathrm{~mm}$ diameter sample grid which had $200 \mathrm{grid}$ bars per inch. The electron beam was constricted by an electromagnetic lens and a transmission diffraction pattern obtained on a fluorescent screen. Film replaced the screen for a trial run and the diffracted beams exposed it through ionization to record the pattern (see Figure 3 ).

A polycrystalline screen was made by fastening aluminum oxide powder to a metal plate using Torr-Seal, a low vapor pressure resin. No pattern whatsoever was visible to the naked eye when this screen was substituted for the fluorescent one even with the laser on. A new screen was fashioned in a similar way using large $1 \mathrm{~cm}^{2}$ slices of single crystal $\mathrm{Al}_{2} \mathrm{O}_{3}$. Again negative results were obtained. The polycrystalline screen exhibited a faint amber glow where the central electron beam impinged. The large sinqle crystals could be made to glow a light blue if bombarded by the entire undiffracted beam. Impurities present in the

'Fused quartz is more transparent to the blue end of the spectrum than ordinary $\mathrm{glass}$ by about $1 \%$. 
Figure 3. Print of the negative obtained by transmission diffraction of molybdenum trioxide 
THIS PAGE

WAS INTENTIONALLY

LEFT BLANK 


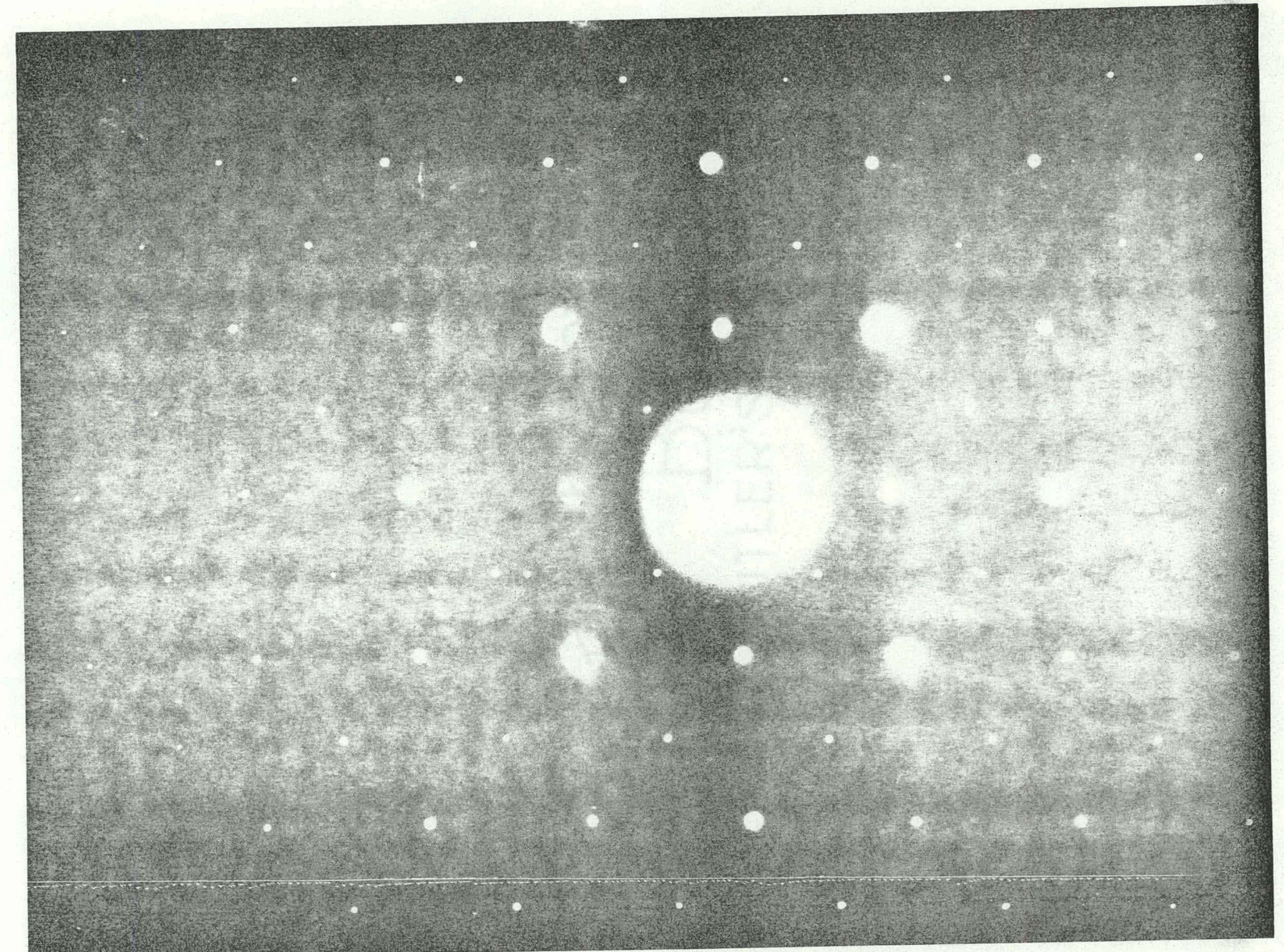


resin were suspected as the cause of the first observation. The blue glow of the large crystals was attributed to the presence of a minute amount of some impurity or a process more complicated than spontaneous emission. Nevertheless no reason could be found for the absence of the Schwarz-Hora effect if indeed the effect did exist.

B. An Analysis and Some New Revelations

In a private communication Dr. Schwarz assured the group that the effect was indeed large and not a threshold phenomenon. He suggested that our apparent failure could possibly have been due to lack of laser power or not sharp enough energy resolution or perhaps our vacuum was not good enough. Schwarz claimed that he had a purple glow from the electron beam present on his nonluminescent target and it impeded his observation of the effect. Not until his vacuum was $10^{-9}$ Torr did he get rid of this glow and his normal operating vacuum was $10^{-10}$ Torr (5). In the preprint previously referenced he pointed out that an ion pump was necessary to achieve such low pressure as well as "baking" of the bell jar which housed the electron optics. Also revealed at this point was the appearance of the effect with the $5145 \&$ laser line. The distance from the screen to crystal was varied between 10 and $25 \mathrm{~cm}$ with the result that the intensity at the target went through minima and maxima. The maxima were spatially separated by about $0.85 \mathrm{~cm}$. This distance apparently decreased with decreasing electron energy. A photomultiplier was used to measure the change in intensity and Figure $4^{1}$ reveals the

\footnotetext{
'Taken from the preprint of Reference 5 .
} 


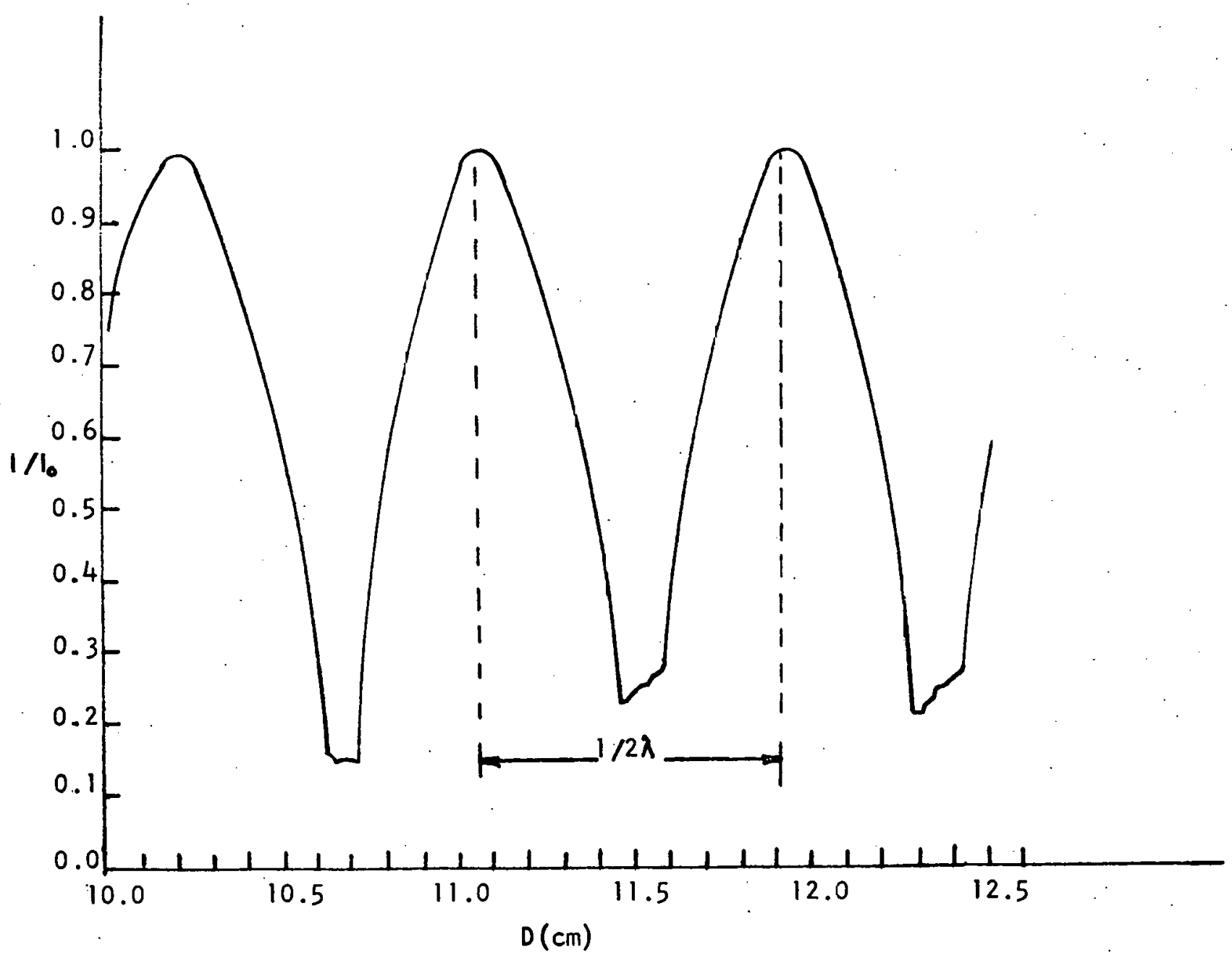

$\mp$

Figure 4. Unities intensity of laser color spots versus crystal to screen distance as measured by Schwartz 
result of the measurement at $50 \mathrm{keV}$. Schwarz also stated that an electron energy resolution of $0.1 \mathrm{eV}$ was necessary for a successful experiment, but he did not claim to have measured hls resolution.

The Schwarz-Hora apparatus was progressively becoming more complicated (5).

An electron gun similar to the one used in the investigation of the Kapitza-Dirac effect served for the production of the $50 \mathrm{keV}$ electron beam of about $0.5 \mu \mathrm{a}$ current and a few micrometers diameter. It was attached to the bell jar horizontally with a varian type flange using a copper gasket. Magnetic and electrostatic lenses and several diaphrams shaped the beam which was bent in the center of the bell jar into a vertical direction. It then passed through three more small apertures before traversing the thin crystalline film to produce an electron diffraction pattern on the screen. The main reason for the horizontal positioning of the electron gun was to prevent scattered light of the hot hairpin filament from shining onto the screen.

Not only was the electron optics considerably more elaborate than previously revealed but also a special lens was used inside the bell jar to focus the laser beam which entered the stainless steel bell jar through a glass window. It was also said that it was necessary to clean the non luminescent target by Ar ion bombardment to reduce background radiation.

A review of our first experiment was in order. We were using a one watt laser compared to Schwarz's 10 watt laser. Our vacuum was four orders of magnitude worse. The latter was disqualified as an impediment as well as the argon ion screen cleaning because we did not experience any difficulty with background radiation on the screen when the apparatus was in the diffraction mode. Bending of the electron beam was not necessary because a series of $50 \mu \mathrm{m}$ to $100 \mu \mathrm{m}$ apertures in the microscope 
prevented any visible light from the filament from reaching the target. Our current was varied from lower to considerably higher than Schwarz's so we had no current limitations. Our electron beam energy was not continuously variable so we could not operate at exactly $50 \mathrm{keV}$. But it was not expected that the experiment should be successful at only one discrete energy. Our energy coherence was uncertain but the electron microscope's optics reasonably approximated that of Schwarz. Energy spread in the electron beam is primarily a result of using a thermionic emitter. Perhaps the angle of the sample holder or presence of grid bars impeded the laser beam. It was also possible that since we did not vary the crystal-to-screen distance that we were situated at an intensity minimum. If we used a more powerful laser, changed the holder geometry, modified the lens system, and also varied the crystal to screen distance, we could surmount the decidedly few obstacles to a successful experiment. Experiment number one turned out to be much less sophisticated and less deliberate than the one to follow. It was attempted because of its low cost and convenient availability of equipment. With the little information available at the beginning an elaborate effort was not possible. 


\section{THE SECOND EXPERIMENT AT I.S.U.}

\section{A. Apparatus}

It was soon discovered that a $10 \mathrm{~W}$ continuous $\mathrm{Ar}^{+}$laser with a $4880 \AA$ output was a nonexistent entity'. With some difficulty and the help of Bell Telphone Laboratories the source of Schwarz's laser was traced to Carson Labs Inc. A representative of the company assured us that a $10 \mathrm{~W}$ laser did not exist but was being planned. He also assured us that Schwarz used one of their lasers for his experiments. It was rated at $2 \mathrm{~W}$ total power for all lines and $1 \mathrm{~W}$ guaranteed minimum in the $4880 \&$ line. Here the first in a series of ambiguities reported by Schwarz was discovered. Arrangements were made to lease the same laser for the next experimental effort at I.S.U.

In order to observe better what was happening with the crystal an elaborate modification of the electron microscope compared to what was previously used was in order. A device called the universal diffraction attachment was mounted in place of a magnetic lens which was not necessary anyway (see Figure 5). A special fused quastz window was ground and mounted using a neoprene o-ring seal in the front of the vacuum chamber to allow passage of the laser beam. The sample holder would be mounted on an arm which could be moved in and out of the chamber in a direction perpendicular to both the electron and laser beam directions. It could also be rotated about the axis defined by that diroction and could bo

\footnotetext{
'Since then they have become commercially available.
} 


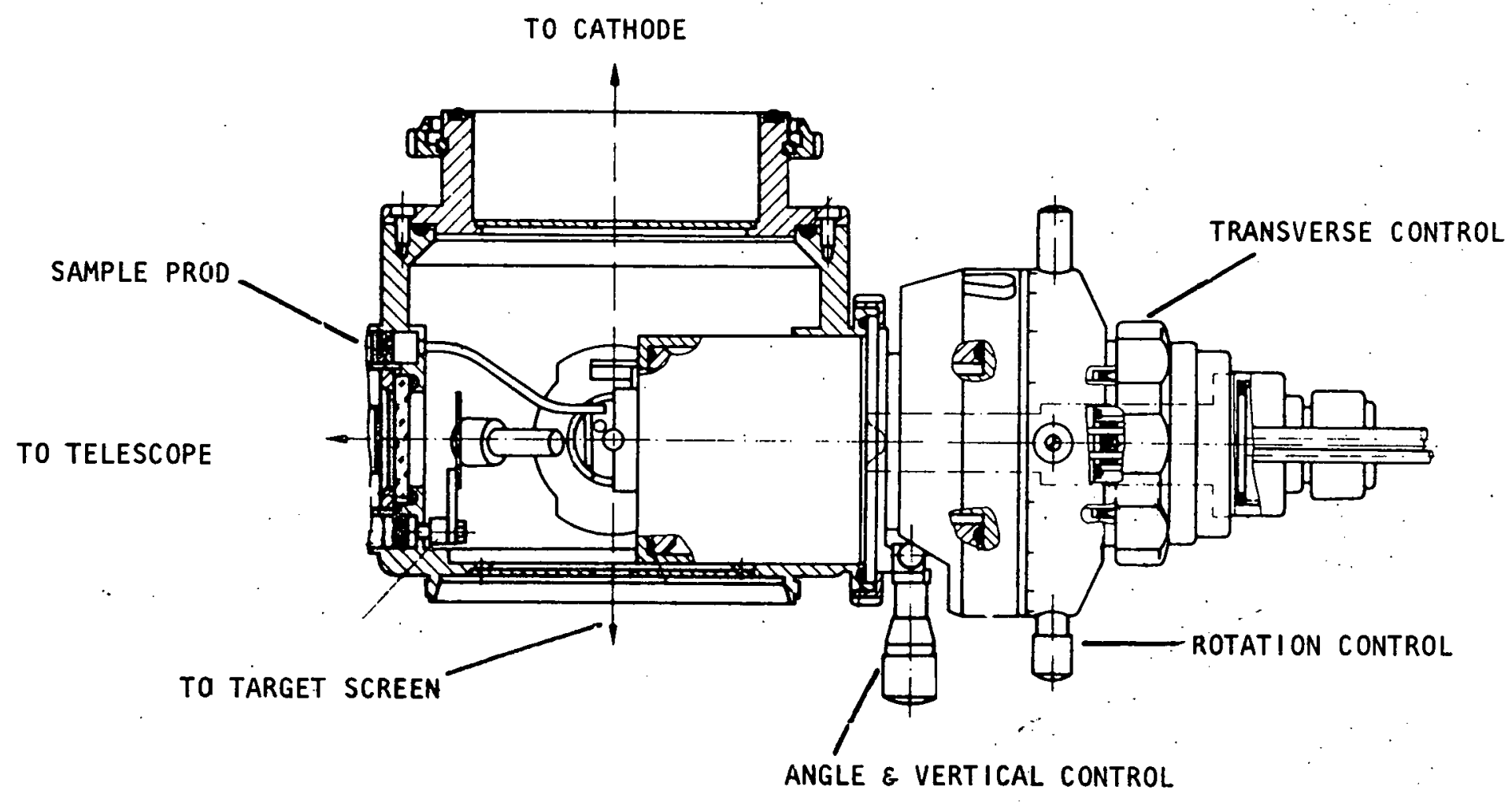

Figure. 5. Universal diffraction attachment 
moved up or down along the electron beam axis. A telescope was mounted on the opposite side to view the sample close up through a window heavily leaded to prevent $x$-ray exposure. Above the telescope was a vacuum sealed rod which could move and manipulate the sample holder and was controlled by an exterior knob.

A special sample holder was devised with a protruding shelf. The thick end of a film would be mounted on the shelf with silicone vacuum grease leaving the other end suspended and accessible to both the electtron and laser beams. A friction fitting was machined on the end of the holder so that it could be inserted into the moveable arm of the universal diffraction attachment. The sample could then be tilted, rotated, or moved in any direction except along the laser axis all without breaking the vacuum.

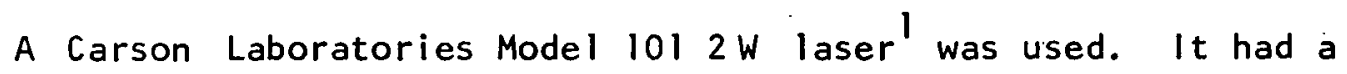
tunable output with two blue lines, two green, and one blue-green. Maximum power of $1 \mathrm{~W}$ was produced in the blue-green $4880 \&$ line, about $1 / 2 \mathrm{~W}$ in the $5145 \&$ line and the other $1 / 2 \mathrm{~W}$ distributed in the other three lines. The laser was equipped with Brewster angle windows which provided a beam polarized in the vertical direction ${ }^{2}$.

The laser optics was quite elaborate. In order to reduce the size of the focus considerably, more than a simple lens is necessary. Because

IThe same one used by Schwarz.

${ }^{2}$ This was the electron beam direction. The subsequent optics did not change the polarization. 
the spreading and subsequent focusing of the laser beam would take up more room than the previous experimental set up it was necessary to send the beam around the room (see figure 6). The beam left the laser with a diameter of $5 \mathrm{~mm}$. It was reflected down and toward the back of the room where it met another mirror. These mirrors were specially coated to give nearly $100 \%$ reflection for light of wavelength $4880 \AA$. From the second mirror the beam was reflected onto a small lens at the back of a collimator. The beam was focused down to less than $100 \mu$ and an aperture to eliminate unwanted diffraction effects was placed at the focus. The aperture was movable in any direction perpendicular to the beam. Past the focus was another large lens system with effective focal length exactly equal to the distance from the aperture to the principal plane of the lens system. The aperture thus served to provide nearly a point source of light for the following lenses facilitating the production of the final wide parallel beam. Emerging from the collimator the beam diameter was $25 \mathrm{~mm}$. This wide beam impinged on the final lens which focused the beam through a quartz window into the vacuum chamber of the universal diffraction attachment. At the crystalline film the laser beam was measured to carry half of its power through a cylindrical region $50 \mu \mathrm{m}$ in diameter and $800 \mu \mathrm{m}$ long. The laser produced $1 \mathrm{~W}$ in the $4880 \AA$ line. Approximately a $4 \%$ loss of power due to reflections is found at every uncoated air glass interface. Ten of these interfaces were present in the system each reducing the power impinging on it to 0.96 of its initial value. The power density delivered within the 


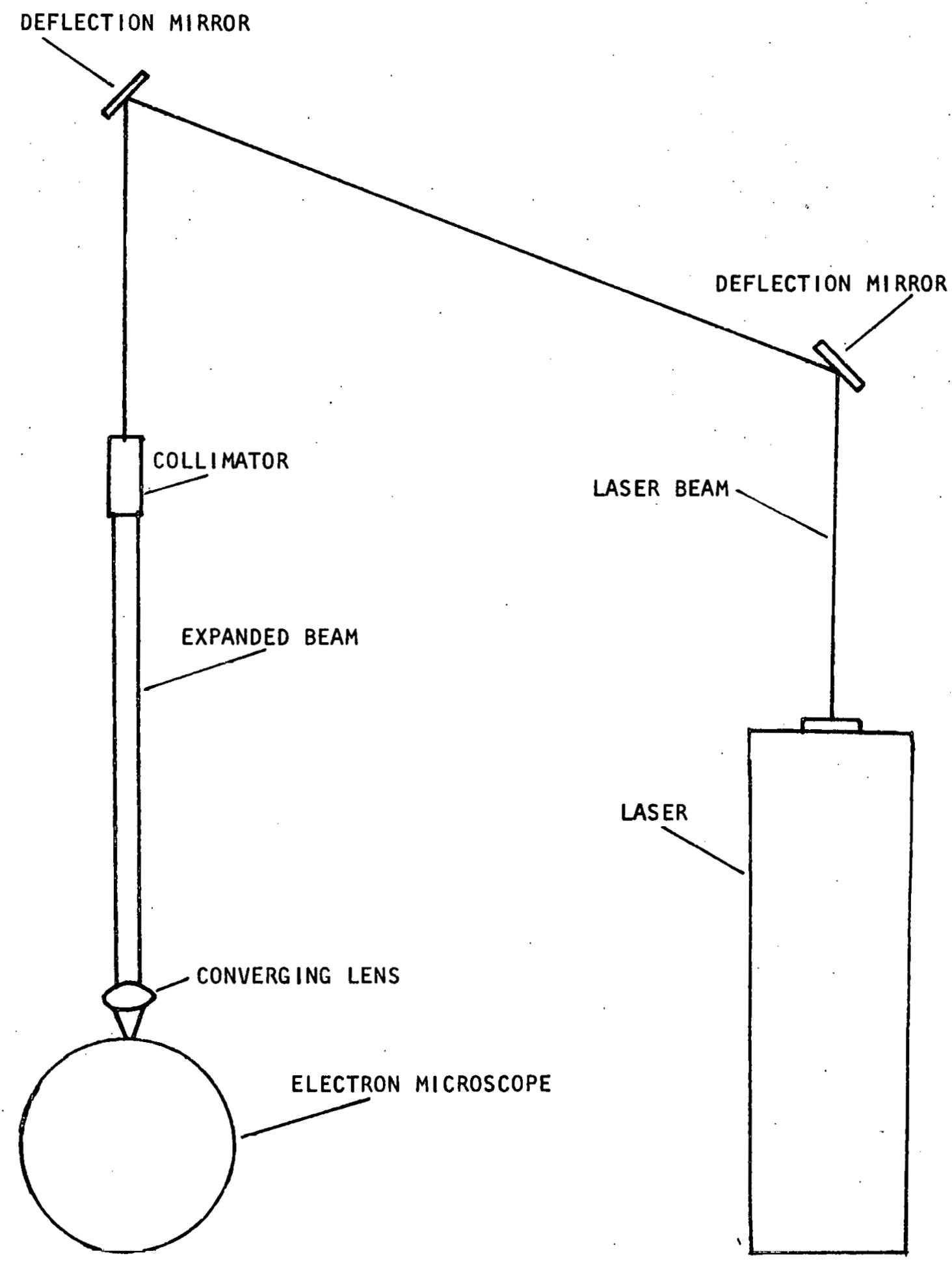

Figure 6: Room layout for the second experiment 
cylindrical region at the film was thus about $1.3 \times 10^{4} \mathrm{~W} / \mathrm{cm}^{2}-a$ sizable increase over the first experiment. 1

The position of the focal region was controllable by a set of micrometers on the final lens mount. One micrometer moved the lens along the longitudinal direction of the laser beam. Another micrometer moved the lens perpendicular to this direction in the plane of the crystal sample. Three more micrometers placed in a triangle about the lens in the longitudinal direction controlled the attitude of the lens. With these last controls the lens could be put in any plane which slightly deviated from the plane normal to the laser beam direction and consequently could be tilted at precisely controllable small angles.

For the electron beam source a hot hairpin tungsten filament was used for most of the experiment although some M-P pointed tungsten filaments were tried. The hairpins produced considerably more current than the pointed filaments. However, they operate at a higher temperature and thus cause a larger uncertainty in electron energy. A series of apertures of the order of $50 \mu \mathrm{m}$ were positioned at various intervals along the microscope column in order to select the most desirable portions of the electron beam. They aiso served to prevent any visible light from the filament from reaching the target area. Two condenser lenses were used to preshape the beam and at times the objective lens was used (see Figure 7). Adjustments could be made in the condenser section so as to

'By more than two orders of magnitude. 


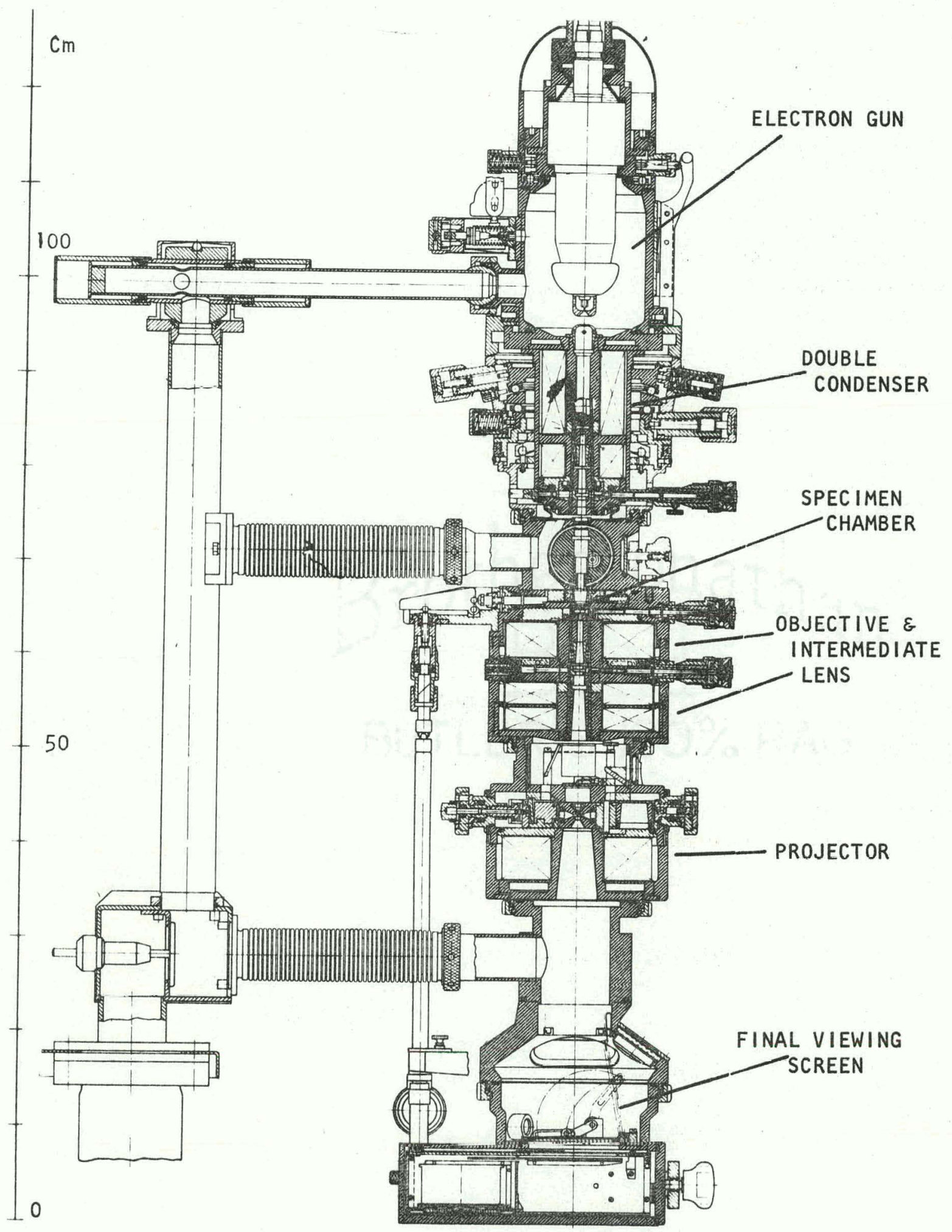

Figure 7. Detailed diagram of the electron microscope with the projector lens in place 
manipulate the beam within the column in order to hit any section of the sample placed on the sample holder.

The target chamber held a fluorescent screen on the bottom which could be lifted by means of an external lever to expose a target below it. The target region below the screen was about $5 \mathrm{~cm}$ tall and targets could be placed at various heights within this region in order to vary the crystal to screen distance. When in operation the electron beam could be located to within $1 \mathrm{~mm}$ on the crystalline film. A transmission diffraction pattern through the thin edge was obtained and the crystal moved into a position and/or slightly rotated so as to obtain a pattern of maximum brightness on the fluorescent $\mathrm{ZnS}$ screen. When the laser. was turned on and focused on the crystal, however, so much scattered light came through the $2.5 \mathrm{~cm}$ diameter hole in the floor of the universal diffraction attachment that the entire target chamber seemed highly illuminated'. This light was mainly the result of the beam scattering from the chamber walls after passing through (and around) the crystal. It had to be eliminated from the target area. First a thin $400 \AA$ thick Al film was suspended on a fine wire mesh over the $2.5 \mathrm{~cm}$ hole. It was thin enough to allow passage of the high energy electrons but reflected essentially all the light incident upon it back into the sample chamber. Although apparently an ingenious idea it was soon abandoned. Faint

'The only light sources in the room were certain control pilots and the laser. Less than one watt of diffused laser light entered the chamber, but to the dark adapted eye it was an unacceptable background. 
transmission diffraction rings were visible on the $Z$ n S screen superimposed over the single crystal mica pattern. Although only a small. fraction of the electron beam interacted with the Al film it was also true that only a small fraction interacted with mica samples used ${ }^{\text {. }}$ The two diffraction patterns were distinct - the mica produced spots characteristic of the single crystal and the Al produced the rings characteristic of a polycrystalline structure. Since a full understanding of the theory was not yet available, it was thought that perhaps some electron demodulation process might occur in the Al film before the beam ever reached the nonluminescent target and thus invalidate the experiment. In order to avoid the critcism of others and our own apprehension, another method not as effective but quite sufficient was developed.

When passing through the crystal sample, the electron beam was only a fraction of a millimeter in diameter. A thick ${ }^{2}$ piece of aluminum foil was placed about two millimeters below the film with a $1 \mathrm{~mm}$ diameter pin hole in it placed directly below the target region. Most of the reflected light from the sample and holder would never pass through the pin hole but neither the electron nor incoming laser beams were obstructed. Since the chamber had to be evacuated the foil could not

'The reasons for using mica will become apparent later in the manuscript.

${ }^{2}$ About ten mils. 
extend over the whole chamber diameter and was restricted to an area of about $2 \mathrm{~cm}^{2}$. Not far below the foil a special baffle was designed so as not to interfere with the electron beam, further prevent scattered light from reaching the chamber below, and yet facilitate evacuation (see Figure 8). Only a small amount of background light was then detectable to the dark adapted eye.

\section{B. Film Samples}

In their attempts to produce single crystal films for their experiment, the group at Bell Laboratories discovered an interesting result. The amorphous film and its substrate are immersed in water. The soapy layer dissolves leaving the thin nonwater soluble film above it which is caught on a specimen holder. It is placed in an electron beam and heated. Reportedly the crystallites align themselves to form a single crystal film. Evidence is seen in watching the diffraction rings of the amorphous substance slowly being replaced by a single crystal Laue pattern. The physics department at I.S.U. possesses the equipment necessary for the evaporative deposition of metallic films but does not have the capacity to produce $\mathrm{Al}_{2} \mathrm{O}_{3}$ or $\mathrm{SiO}_{2} \mathrm{films}$. So other methods of crystal growing needed exploration. Crystal production by use of supersaturated solutions was an area of technology familiar to the author for many years. Attempts were made to grow small crystals of sodium nitrate and rochelle salt. The hydrate of each forms a nice clear crystal which would not hamper the passage of a light beam. A solution is made by dissolving the material in water near the boiling point. The solutions are allowed to 


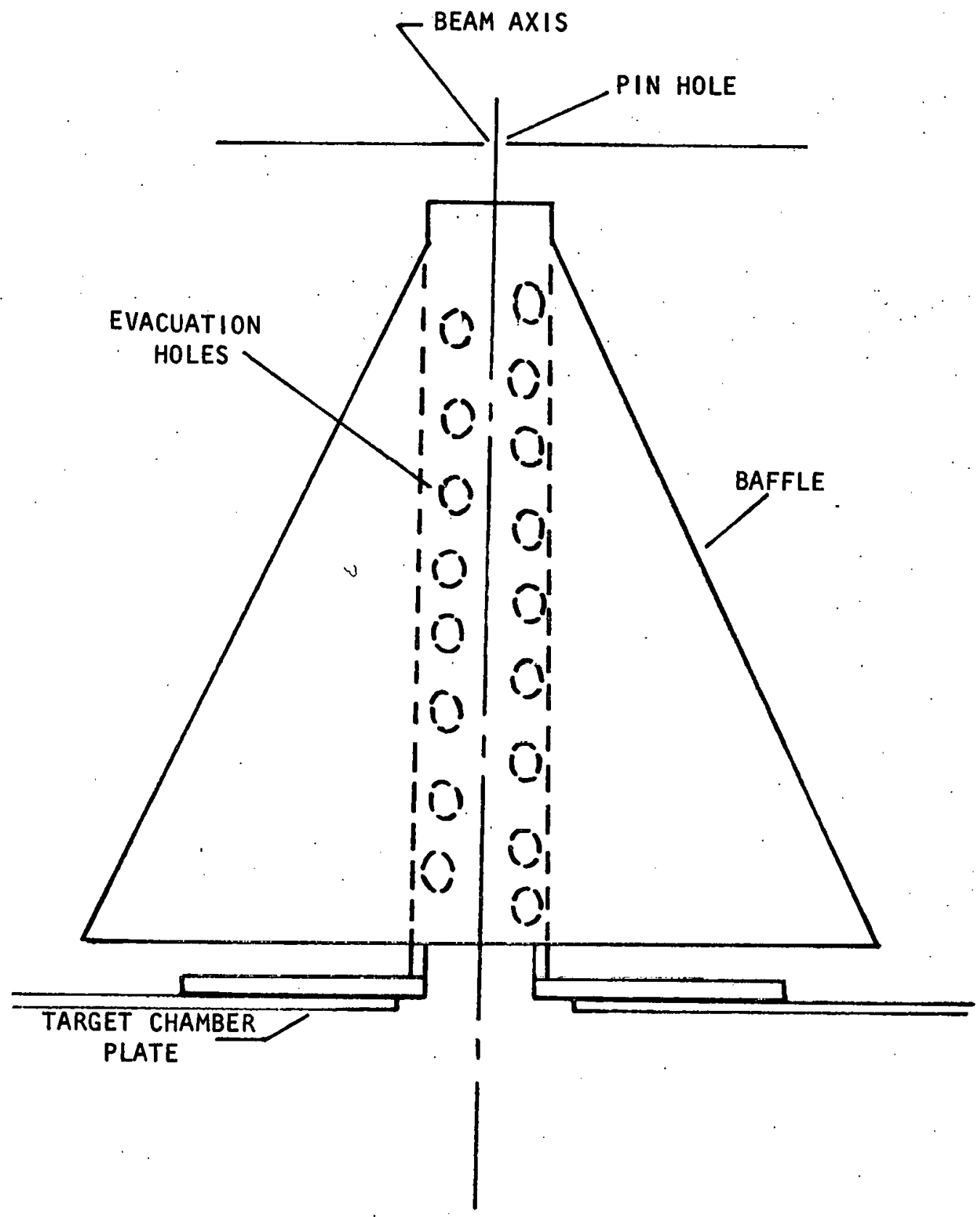

Figure 8. Baffle permitting passage of the electron beam and easy evacuation yet obstructing the passage of light into the target chamber 
slowly cool in a constant temperature room free from disturbing vibrations. ' A few crystals form on the vessel walls and a large number through evaporation float on the free surface. Upon reaching a size sufficient enough so that their weight overcomes the surface tension of the liquid, they descend to the bottom and continue to grow. The rochelle salt crystals grow larger and faster than sodium nitrate. But thin films of these crystals were needed. Cleaving was thought appropriate to produce a thin cross section upon which a drop of water could be placed. A portion of the crystal would redissolve. Removal of the solution droplet would leave a (hopefully) thin film. Rochelle salt proved difficult to cleave and was discarded. The sodium nitrate was ideal. However the redissolving process proved too clumsy to produce a nice film.

It was then discovered that by use of a magnifying glass and careful observation a film could be found still floating on the solution surface. Films easily visible could be lifted off the surface by placing a submerged specimen holder beneath it and gently bringing both up and out of the solution. These crystals were too thick to allow transmission electron diffraction. Next it was found that a crystal too thin to show a definite outline could be located by the interference colors it produced. These again were too thick. Next, places on the surface could be located where a slight well appeared but no crystal outline or

Tiny crystallites form from evaporation at the surface. The smallest wave propagating across the surface of the solution causes some microscopic crystallites to fall from the surface and through the solution. They serve as thousands of tiny nuclei upon which so many larger crystals grow that they obstruct each other and become useless. 
interference colors. In the shallow well lay a very thin film. About $80 \%$ of these were destroyed in trying to mount them on a specimen holder. of those that survived mounting none survived the heat produced by electron bombardment for more than several seconds. A diffraction pattern would appear and suddenly the crystal would evaporate. Sodium nitrate could not survive in the vacuum upon heating and a further search could not find a water soluble salt that could. Liquid nitrogen cooling of the specimen was possible with a special sample holder. Inevitably, however, some part of it would obstruct passage of the laser beam and could not be tolerated.

A substance which could withstand considerable heating was sought and mica because of its cleavage properties was a likely prospect. Cleaving mica to the useable thickness of only a few thousand angstroms with a macroscopic instrument appeared to be an impossible task.

But a way was found to do it. Reasonably thick slices of the material were put into an ion mill. High velocity Ar ions bombarded the mica and slowly ate away the surface. Unfortunately a rough surface as seen through a microscope resulted and it would add to the difficulty of understanding the laser field geometry at the surface of the film. Another impediment was the deposition of layers of carbon on the film surfaces. 0 il vapor from the diffusion pump in the machine strayed in 3porsc but apparently sufficient quantities to be broken down by the ion bombardment in the target chamber. Resulting carbon molecules attached themselves to the film surface posing the problem of their removal. 
However, another subtle but very successful method was discovered. Cellophane tape was attached to the mica surface and gently pulled off. Several distinct layers would adhere to the tape and very thin portions were sometimes found to overlap the edge (see Figure 9). These sections were removed from the tape by means of a tweezer and stored in a jar suspended from a smaller piece of tape in order to avoid damage. When ready for use a sample was observed under a light microscope at only about $100 \times$ (see Figure 10). Interference colors were seen in various sections with their own somewhat random geometry. Specimens were sought which had a thin clear protrusion on an unobstructed edge. These regions were too thin to exhibit interference colors with illumination at normal incidence. Therefore they had to be less than $1 / 4$ the wavelength of the illuminating light inside the crystal. The index of refraction of mica is about 1.6 and the shortest wavelength of visible light is about $4000 \AA$. For a film thickness d we should have:

$$
d<\frac{1}{4 \mu}(4000 \AA)
$$

or

$$
d<625 \AA
$$

After the experiment one sample was sent to Bell Labs and kindly measured by $L$. Pfeiffer. A sophisticated $\alpha$-particle energy loss technique measured the sample thickness as $3200 \AA$. There is some doubt as to whether the small thin edge survived shipping. Although $3200 \AA$ is thin 
Figure 9. A mica tape peeling sample

Figure 10. Diagram of a $100 \times$ view of a us ab le sample 
THIS PAGE

\section{WAS INTENTIONALLY \\ LEFT BLANK}



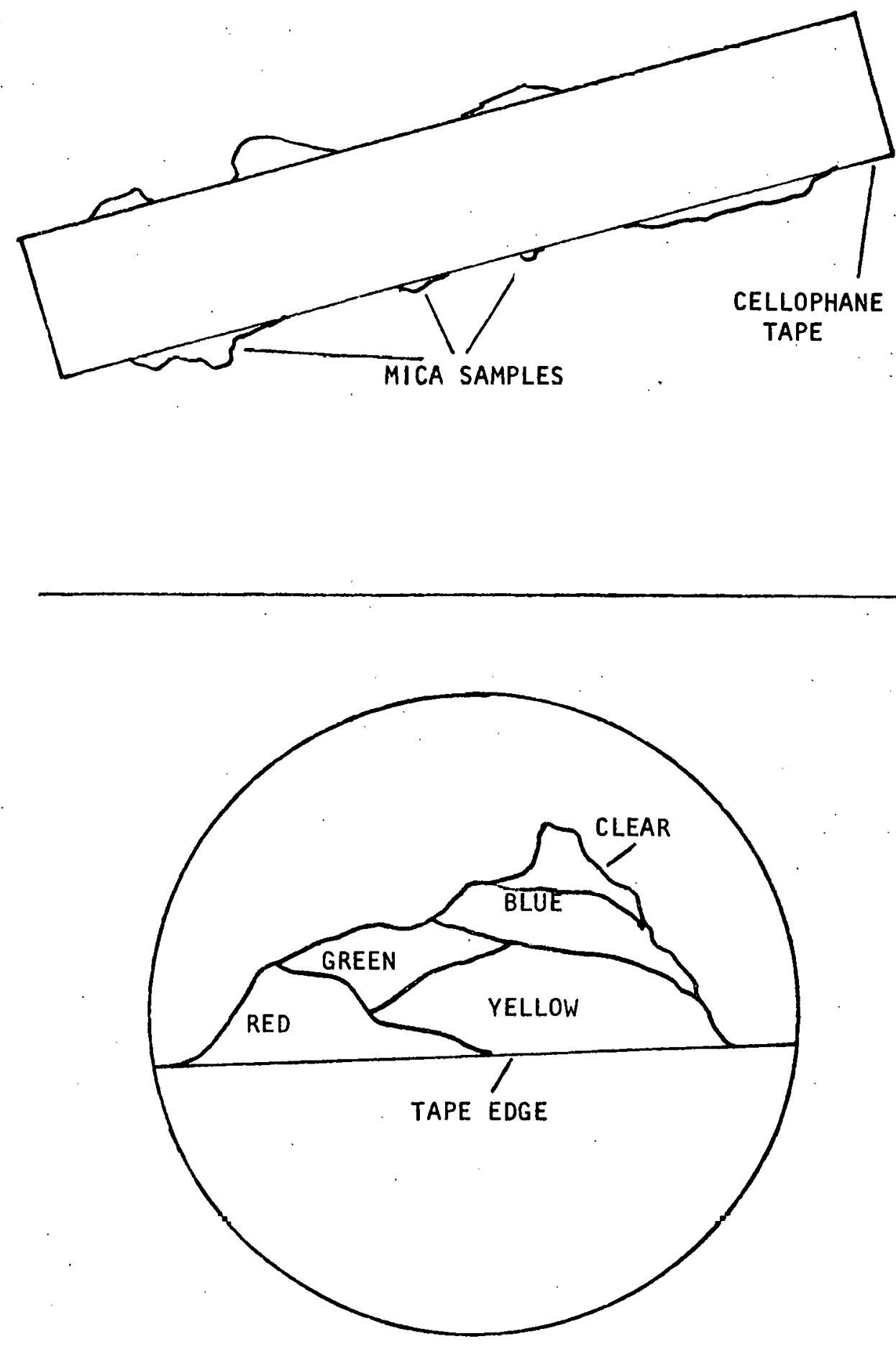
enough to obtain a good diffraction pattern it certainly would have displayed interference colors.

\section{A Final Modification}

Most design problems appeared to have been solved when another one materialized. Everything seemed operational but within a few minutes of turning on the electron beam the diffraction pattern would wander, disappear, and reappear intermittently. The cause was soon discovered. Mica is a dielectric. Obvious is the fact that under a continuous bombardment by electrons the crystal would pick up a static charge. It must be remembered, however, that the crystal is at most a few thousand atoms thick supported by its own strength from a ledge in empty space empty except for the stream of electrons. As the crystal charged it would interact with the electric field of the beam and bend one way or the other. When a sufficiently high density of charge was reached it would discharge (probably to the sample holder) and return to its initial configuration. The film might be said to have been flapping in the electron breeze - for this is how it appeared - with a period of about one second'.

A means was soon adopted for the prevention of sample charging. Exactly opposite the window where the laser beam entered the sample chamber a port was opened. An "auxiliary" electron gun was mounted in

IAfter an initial charging period of a few minutes. 
this port and extended out to near the sample. A special shield with only a small hole in it covered the filament so as to prevent excessive illumination of the chamber. The auxiliary gun was supplied with $3 \mathrm{ma}$ of current at $300 \mathrm{~V}$. Residual gas molecules in the vacuum chamber were ionized thus allowing the sample to discharge. The "flapping"! problem was solved and no more major obstacles were encountered in operation of the apparatus.

\section{Procedure}

When a sufficient vacuum was obtained within the microscope ${ }^{l}$ the electron beam was switched on and in the magnification mode the sample was observed by its electron shadow on the nonluminescent screen. A thin portion on the edge would be located and the mode changed to give a diffraction pattern. By alternately viewing the sample shadow and looking at the diffraction pattern an ideal place on the crystal could be located which would be accessible to the laser beam and produce 5 to 50 diffraction spots. From the edge a slight luminescence could be seen in the crystal where the electron beam was incident. At this point the beam was 10 to $20 \mu \mathrm{m}$ in diameter. The laser was focused down and the focus positioned as near the electron beam crystal intersection as could be determined by eye. The only lights left on were instrument lights for monitoring meters. Black hoods were draped over observers and target.

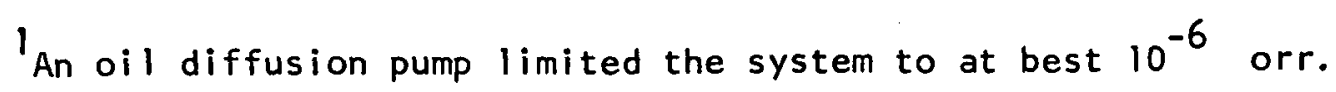


chamber so the lights would not be distracting'. Approximately 10 ininutes was allowed for an observer's eyes to become accustomed to the darkness. Scanning was then begun by moving the laser focus in steps of about $15 \mu \mathrm{m}$. It was first moved in the plane of the crystal until a sweep through the electron focus plus some extra was made. Then it was moved perpendicular to the crystal plane and a sweep back made. All this time the observer watched the nonluminescent target and reported any visual observations ${ }^{2}$. A faint amber glow in the central spot on the nonluminescent target was usually all that was visible and increased and decreased in intensity depending on electron current, position of focus, and the psychological factors in the human eye. If it turned out to be an obstacle in viewing the $4880 \&$ wavelength, observation would have been made through a $4880 \AA$ interference filter which would have blocked all spurious wavelengths. The use of this filter was never necessary.

The crystal to screen distance was varied in steps of $5 \mathrm{~mm}$ by placing or removing film holders from the airlock beneath the target screen. Distances between 30 and $34 \mathrm{~cm}$ were used. No effect was observed and other target materials were tried. A sheet of mica was tried in case, for some unknown reason, some type of coincidence was needed between

Ihe effect was supposed to be easily visible - but to observe it on the threshold of visibility before all parameters had been maximized was desirable.

2Wich usually turned out to be no more than hallucinations. After a long period of time in the total darkness an intent observer might faintly see shapes and lights which were no more than mental images. 
crystal and target material or structure. A Schott 0G-2 filter and a sheet of copper were also tried as target materials because they absorb $4880 \&$ light and hence can have $2.54 \mathrm{eV}$ excitations. These were used to check out suspicions that the target needed: resonances in the $2.54 \mathrm{eV}$ region in order to produce the blue light. sought. This would have been contrary to Schwarz's report but it was thought that perhaps his target was inadvertently doped with some contaminant. For each target material all approprlate parameters were varied and enough scans made to insure the intersection of the laser and electron beams. Instead of a continuous scanning procedure a delay of about $10 \mathrm{~s}$ was left in between movements of the micrometer setting in case there was some delay time necessary to bring the effect to visibility. Still nothing was observed.

The importance of field discontinuity at the modulating crystal was tested by using a $400 \&$ thick Al film. The metal should produce a sharper discontinuity than the dielectric in the electric field of the laser beam. Diffraction rings were observed on the $\mathrm{ZnS}$ screen but again nothing on a nonluminescent target. There was no reason to expect that the amorphous structure should greatly impalr the modulation process' single crystals were originally used because the best possible duplication of the original Schwarz-Hora apparatus was sought.

With the lack of success in the transmission diffraction experiments, reflection diffraction was tried. Macroscopic samples of $\mathrm{NaCl}$ and $\mathrm{Mg} \mathrm{O}$ were mounted on a special sample holder so that one side was approximately

'Agreed to even by H. Schwarz (Rensselaer Polytechnic Institute) in a private communication. 
where the thin film edge was in the sample chamber. This side was not more than a few degrees out of the plane made by the intersecting laser and electron beams. The electrons were set at grazing incidence to the sample. The laser was focused down to about $0.25 \mathrm{~mm}$ diameter. Scans were made and in each case only electron diffraction was observed on the fluorescent target.

\section{E. An Analysis of Results}

It appeared as though either something was fundamentally wrong with. the experiment or the Schwarz-Hora report. Lack of electron current or laser power were first considered again. The electron beam heated thick portions of the sample to incandescence but it did not glow when the beam passed through the thin portion. It therefore appeared possible to increase the beam current without destroying the sample. However, the currents used ranged more than an order of magnitude higher than that reported by Schwarz and therefore no benefit could probably be gained by going still higher especially since greater currents increased charging problems and decreased filament life ${ }^{l}$. It was not known exactly what Schwarz's laser intensity was at the sample and he made no indication of an attempt to measure it. Our operating laser power density was $1.3 \times 10^{4} \mathrm{~W} / \mathrm{cm}^{2}$ delivered at the sample. With a different $\mathrm{Ar}^{+}$laser and special power meter it was determined that an intensity exceeding $1.5 \times 10^{4} \mathrm{~W} / \mathrm{cm}^{2}$ led to serious damage or total evaporation of the mica

'A chief expense in operation of the electron microscope. 
samples.' So the power could not be raised substantially.

Again, a number of conclusions were reached in light of failure to observe the effect as reported in Reference 4:

1) There may be a necessity for some subtle geometry involving the crystal and both beams. Until more is understood, however, there. can be only mystery.

2) Our electron coherence length may not have been correct. We were unable to change it. The energy spread in our electron beam was estimated to be about $0.7 \mathrm{eV}^{2}$ Schwarz in Reference 5 suggested that his spread was about $0.1 \mathrm{eV}$ but he made no description of how it was estimated. Our higher current and beam energy may have compensated.

3) Laser power may have been inadequate. Although the mica samples could not withstand a greater power density than we used, the $\mathrm{Al}_{2} \mathrm{O}_{3}$ and $\mathrm{SiO}_{2}$ which Schwarz used have melting points of $2050^{\circ} \mathrm{C}$ and $1710^{\circ} \mathrm{C}$ respectively and can withstand higher temperatures than mica with a melting point at about $1300^{\circ} \mathrm{C}$. However, Schwarz also reported (5) using $\mathrm{SrF}_{2}$ which has its melting point at $1190^{\circ} \mathrm{C}$ and clearly is more

'They were observed through a telescope with laser power monitored by a meter immediately preceding the sample mounted in the atmosphere. Consistently damage occurred when the power density even slightly exceeded $1.5 \times 10^{4} \mathrm{~W} / \mathrm{cm}^{2}$.

${ }^{2}$ The Siemens Corp., Iselin, N.J. who bullt and maintain the electron microscope estimated the energy spread to be 0.4 to $0.5 \mathrm{eV}$ from the thermionic emitter. Additional spread of up to a few tenths of an $\mathrm{eV}$ may result from electron-electron scattering at the foci of the magnetic lenses (The Boersch Effect). 
susceptible to heat destruction than mica. Also Schwarz reported seeing the effect with the green line of the $\mathrm{Ar}^{+}$laser and the red line of a $\mathrm{Kr}^{+}$laser (14) both of which are considerably weaker than the $4880 \&$ line originally used. If the color of the spots was observed as reported by Schwarz then an effect $1 / 500$ as intense would be visible to the unaided eye but with undeterminable hue (15). We saw nothing.

4) The effect for some unknown reason may be weaker in mica than in $\mathrm{SiO}_{2}, \mathrm{SrF}_{2}$, and $\mathrm{Al}_{2} \mathrm{O}_{3}$ or perhaps the mica sample thickness was not optimum. The implications of sample thickness have been theoretically reviewed and will be presented in the second half of this manuscript. 5) The target screen was not glow discharge cleaned as Schwarz's was. However, his purpose was to eliminate background radiation which never posed us a problem.

6) The effect may be much weaker than reported' or not exist at all.

'Too weak for photoelectric observation as well as visual observation from results at 1.S.U., Bell Labs, Brooklyn Poly. 


\section{DISCUSSION}

Since the intensity of the effect is somewhat of an important question, a slight revlew of the eye as a perceptive optical instrument may be appropriate. Schwarz estimates the total radiant power from the spots on the nonluminescent screen to be about $10^{-10}$ watts (5). It must be remembered that this is not a measurement but an estimate. The human eye is a delicate instrument with an enormous responsive range which has been painstakingly studied by physiologists and psychologists. Studies made by M. H. Pi renne have been published in Reference 15 . Chromatic or photometric vision is conducted by the cones on the retina with sensitivity to light of all wavelengths in the visible spectrum under normal daylight intensities. This color perceptibility stops at a threshold about 500 times higher in intensity than the minimum intensity of perceptibility for an eye in darkness. As incident intensity is decreased, the rods on the retina take over the job of perceiving and the cones become nonfunctional. The rods, however, are not sensitive to wavelength ${ }^{\prime}$ and can only achromatically ${ }^{2}$ perceive. The eye becomes more sensitive to small amounts of 11 ght as time allows it to adapt itself in a completely dark environment. After approximately 10 minutes the curve relating minimum perceptible intensity to time adaptation begins to level off and is essentially flat from 30 minutes upward.

'They do not communicate to the brain which color is being observed.

${ }^{2}$ Things are either black or white. 
Pirenne reports that the dark adapted eye can perceive a source with a minimum brightness of $10^{3} \mu \mu \mathrm{L}$ at a distance of one meter. Here one Lambert is the unit of brightness equal to $1 / \pi$ candle per $\mathrm{cm}^{2}$. If the target brightness is the minimum acceptable of $10^{-9} \mathrm{~L}$, then each $\mathrm{cm}^{2}$ of visible target must radiate $10^{-9} / \pi$ candles. An intensity of one candle produces a lumen per $\mathrm{ft}^{2}$ of detector area at a distance of one ft from the radlating source. If the retina is the detector and has the generous area of $1 \mathrm{in}^{2}$, and since a lumen is equivalent to 0.00149 watts, each $\mathrm{cm}^{2}$ of source (the target) must radiate:

$$
\begin{aligned}
\text { Power } & =\frac{10^{-9}}{\pi} \text { candles } \times \frac{\text { lumen } / \mathrm{ft}^{2}}{\text { candle }} \times \frac{0.00149 \text { watts }}{\text { lumen }} \times \frac{\mathrm{ft}^{2}}{144 \mathrm{in}^{2}} \times \\
& \times 1 \mathrm{in}^{2} \text { (of retina) } \\
& \approx \frac{10^{-14}}{\pi} \text { watts. }
\end{aligned}
$$

To observe color an increase of intensity by a factor of 500 is needed to bring the radiated power per $\mathrm{cm}^{2}$ to about $10^{-12}$ watts. Now if observation is to be made from across the room, say at $10 \mathrm{ft}$ away Instead of 1 $\mathrm{ft}$, the needed power turns out to be about $10^{-10}$ watts - Schwarz's estimate. However each $\mathrm{cm}^{2}$ must radiate this power and still only a threshold effect is believalle. If the eye were not dark adapted several orders of magnitude would also be needed to compensate. At the threshold of 
observation, the eye may not see a continuous image but rather one that fades into and out of visibility periodically. One is led to the conclusion that either Schwarz's power estimate is off by several orders of magnitude or that contrary to his statements the Schwarz-Hora effect is not a threshold phenomenon.

So much mystery still surrounds the apparently observed effect because no researcher other than Schwarz has even seen it in operation. A technician and the head of the physics department at Rensselaer Polytechnic Institute have reportedly observed the blue spots but did not witness a turning off of the laser or any other associated test to lend credence to the experiment. Heinrich Hora, the co-author of the Applied Physics Letter for which the effect was named, has never seen the effect but only the apparatus which Schwarz claims to have used. ${ }^{l}$ The group at lowa State had been refused admittance to observe the effect in Schwarz's lab twice. The first reason given had to do with patent considerations. The second time the apparatus had been dismantled. Likewise the people at Bell. Labs have been refused because the Schwarz lab was being moved to a new building. Scientists have obtained admittance to see the Schwarz apparatus but none have seen it operating.

The results of four experimental groups in the United States trying to reproduce the Schwarz-Hora effect are now known. The lowa State Experiment is the only one published and is reported in detail above. A

\footnotetext{
'Private communication with H. Hora in Garching, Germany.
} 
group at Wayne State University used a laser not as powerful as the other groups and has not observed the effect.' Other details of the experiment are not available. At Brooklyn Polytech, Donald Scarl, Gordon Gould, and Larry Silverstein used a $36 \mathrm{kV}$ electron beam with monochromaticity of about $2 \mathrm{eV}$. The laser delivered $30 \mathrm{~W}$ at the thin film in 10-nanosecond pulses. The thin films were made of polycrystalline aluminum oxide, amorphous quartz, and single crystal mica. Negative results were obtained (16).

At Bell Labs Loren Pfeiffer and Denis Roussea used a $100 \mathrm{kV}$ electron gun which was mostly operated at $50 \mathrm{keV}$. The monochromaticity was limited by cathode temperature. The whole system was contained in a $10^{-9}$ Torr vacuum. The screen was high-purity pressed alumina. A $1200 \AA$ silicon dioxide film of good optical quality was used. Their laser produced one half watt at $5145 \AA$. The results were negative.

The largest criticism of the credibility of any of the experiments including Schwarz's lies in the necessary energy resolution of the electron beam. Schwarz's first estimate (4) was that $0.1 \mathrm{eV}$ was necessary for a $50 \mathrm{kV}$ beam to be successful in producing the effect. In the June 1971 issue of "Physics Today" Schwarz critizes the attempts of all others (16).

Schwarz points out that none of the experimenters are using an energy resolution as small as the value of $0.05 \mathrm{~V}$ predicted by the theory.

I Private communication with P. M. Fradkin, Wayne State University, Detroit, Michigan. 
immediately following the publication of the I.S.U: results in Applied Physics Letters (1), Schwarz was allowed a rebuttal to our criticism in which he says (17):

The main prerequisite, vis., low electron energy spread, to reproduce the effect was, by far, not fulfilled by Hadley et al.

He then points out that this resolution is indeed an issue in describing part of his procedure.

As reported, the monochromaticity of the electrons was spoiled deliberately causing the modulation to disappear.

The Schwarz apparatus is defended with the statement that his energy resolution was an order of magnitude better than his original estimate and five times better than what he said was necessary in "Physics Today."

The energy spread due to the temperature of the pure tungsten fil lament was about $0.1 \mathrm{eV}$, which, after slowing the electrons to $10 \mathrm{eV}$, was further cut down with a velocity analyzer to less than $10 \mathrm{mV}$.

The simple bend which Schwarz originally used to eliminate cathode filament light from the target area had by this later revelation evolved into an elaborate energy analyzer. His first claim was that his 2 in radius bend of the beam was a velocity selector. It was pointed out to him that a $50 \mathrm{kV}$ beam could not be monochromatized with a 2 in radius $90^{\circ}$ bend but would require one on the order of $2 \mathrm{~m}$. His counter argument was quoted above where he said that the beam had been slowed from $50 \mathrm{kV}$ to $10 \mathrm{eV}$, bent, and then sped back up to $50 \mathrm{kV}$.

Assuming this later description to be more accurate than previous ones, the group at Bell Labs published (18) a defense against the attack 
on the I.S.U. group made by Schwarz. It explained how if Schwarz's

latest description of the apparatus was correct and the beam was adequately energy selected, then the surviving current would be orders of magnitude lower than that in Schwarz's report and even so would be the best ever attained in the history of electron beam technology and thus should have been published as a feat in itself. An excerpt from the conclusion reads as follows:

This calculation of the upper limit for Schwarz's beam current is based on the fragmentary accounts of his apparatus that have appeared in his publications. The re are, however, many designs reported in the extensive literature on electron monoch romators that are theoretically more efficient than the one described by Schwarz. The beam current even in the best of these monochromators, however, when operated at $10 \mathrm{MeV}$ resolution, is still limited to about one nanoampere. Since Schwarz has stated several times that his original motivation for deflecting the beam was to prevent light emitted from the cathode filament from shining onto his diffraction target, it is remarkable that his monochromator apparently exceeds the state of the art by several orders of magn i tude.

of course the controversy could not stop here. Schwarz was allowed to defend himself once again and immediately following the above paper Schwarz published (19)

The apparent incompatibility of two requi rements, namely, extremely narrow energy spread of the $50 \mathrm{keV}$ beams of less than $10 \mathrm{MeV}$ and relatively high electron currents of 0.5 pa is overcome by shaping the electron beams into a "filamentary" structure while passing through a magnetic monochromator. This is achieved with diaphrams having many equally spaced holes of diameters as small as $5 \mu$. Proper adjustments of the spacings and of the characteristics of the monochromator provide for zero phase difference between the single electron beam filaments.

His diaphram had $10^{4} 5 \mu$ holes in it which he claimed produced $10^{4}$ times as much current as a single beam could. The argument breaks down theoretically when one considers the proper phase adjustment of the $10^{4}$ 
holes in it. The details are unimportant for their complexity far surpasses the subjective usefulness of staying one step ahead of Schwarz's newly-revealed technical prodigies. With considerable difficulty it may be possible that the multi-hole diaphram could increase useful current by $10^{2}$. However, the complexity of adjusting the parameters to provide zero phase difference for all the beams simultaneously would probably require a computer with monitoring devices amounting to the complexity of a telephone switchboard for an average sized clty. As of this writing Schwarz has made no claim to havlng used such a labyrinth.

The Schwarz-Hora effect has aroused the curiosity of many researchers around the world whether or not it exists. Many papers have been written which reflect the dubious attitude of researchers to the experimental report of Schwarz. Still the theoretical interest spurred by the original paper in Applied Physics Letters has led to interesting calculations previously unconcelved and areas of thought formerly unexplored. The answers which govern how much practical information will eventually be obtained from this area of study ironically will lie with the theorists. All experimental groups in this country have ceased activities in this area. However there is still considerable interest in other countries as evidenced by the dozens of reprint requests received by the author over the past few months. The next section of this manuscript involves a theoretical calculation' which explains why the Schwarz-Hora effect would be at least more feasible, if nul pussible, at higher energy and çontains the complete relativistic calculation of the modulation mechanism necessary for the effect.

IPart of which has been submitted to the Journal of Applied Physics. 


\section{SYNOPSIS OF THEORETICAL PROGRESS AND MOTIVATION FOR CONTINUED WORK}

\section{A. Theoretical Advancements}

During the experimental activities described in the preceding chapters waves of theoretical papers on the Schwarz-Hora effect began to arise. At Wayne State L. Favro, D. Fradkin, P: Kuo, and W. Rolnick worked in varlous combinations with each other to publish a number of papers $(20,21,22,23,24)$. Their works center around the idea that the effect involved electron-electron phase correlations. The modulation of one electron's wave function caused the induced dipole moments in the nonluminescent screen to start oscillating, and they oscillate in phase with the next electron's wave function. The two electrons cooperatively excite the screen and look like four electrons instead of two. Since the effect would be coherent, the group of particles would radiate proportional to the square of the number of particles. The Wayne State group predicted cross-section enhancement but not the absolute level of radiation. These ideas were contrary to those of Hutson (9) who made some observations of the published photographs (4) of Schwarz. He points out that the ratio of the intensity of the central spot to the diffracted spots appears to be approximately the same for the fluorescent target as well as for the nonfluorescent target. For any coherent radiation process the intensity ratio on the nonfluorescent screen should go as the square of the intensity ratio of the pattern on the fluorescent screen, and this can be ruled out by visual inspection of Schwarz's photographs, says Hutson. As for the abillty of the Wayne State theories to adequately 
explain the visual intensity at the target--it was pointed out to Fradkin at a colloqui um at lowa State University that some type of $2.54 \mathrm{eV}$ resonance would have to be present in the target in order to come up with a substantial intensity. Without the resonance the theory would be deficient by more than ten orders of magnitude.

Becchi and Morpurgo from the University of Genoa proposed a many particle quantum mechanical model (25) of the demodulation mechanism and presented a proof that only with this type of demodulation can any substantial intensity be obtained. Using Schwarz's parameters, however, they point out that the intensity predicted at best would fall short of Schwarz's estimate by four orders of magnitude. Other espousers of a many-electron theory were Marcuse $(26,27)$, Kondo $(28)$, and Lipkin and Peshkin. (29). Their work shows rather conclusively that a many-electron effect is necessary. It therefore would remain a mystery, as Hutson pointed out, that Schwarz's photographs give no evidence of such an occurrence.

The ideas on the modulation mechanism proposed by Hadley, Stanek and Good, Jr. in Reference 2 have the alternative interpretations as being either single or many-electron depending on which view the reader chooses. The calculated probability density can be considered a statistical entity assigned to a single particle or may be interpreted as a distribution applied to a large number of particles. The demodulation mechanism proposed circumvents the need for exact understanding of the quantum mechanical happenings within the target. A modulated classical current is used to come up with a generous upper limit for the 
radiated intensity. This calculation was the first and only one published which actually comes up with an explicit expression for the amplitude of the modulation dependent upon Schwarz's parameters and a numerical estimate of this amplitude. The subsequently predicted intensity at the target falls short of Schwarz's estimate by four orders of magnitude- in colncidence with Becchi and Morpurgo's estimate from a totally different point of view.

With the lack of supporting experimental evidence, the lack of cooperation by Schwarz, and apparently the existence of theoretical predictions which contradict Schwarz's reported observations, the motivation for continued theoretical interest might be questioned. There is reason to continue work in this area. The $2 \%$ modulation amplitude found in Reference 2 shows that something does happen to the electron beam. At sufficiently high currents a relatively sizeable number of electrons receive the frequency modulation. A detailed wave packet treatment on exactly what happens in an understandable way is presented by Hadley (30). What remains most questionable is the possibllity of using a nonluminescent target as a detection mechanism for the modulation. Newly-proposed innovations may make Schwarz's mechanism unnecessary. Schmieder has suggested the use of electron interferometry techniques (31). Two separate modulating crystals would be used along with two separate lasers. The lasers would be closely tuned and their beat frequency rather than an optical frequency would be measured. If new detection mechanisms such as this one are feasible, then the only impediment to the production of the FM electron waves is the energy resolution criterion. A more detailed 
discussion of this criterion may be appropriate here.

\section{B. Coherence Criterion}

It is more realistlc to consider a free electron as a localizable entity rather than as a plane wave in studying the relations between two electrons. Let us postulate that the wave packet is gaussian. in shape along a one-dimensional $x$-axls. ' Then the Heisenberg Uncertainty Principle ${ }^{2}$ reduces to $\Delta p \Delta x \simeq \hbar$, where $\Delta x$ and $\Delta p$ are the uncertainties in the position and momentum respectively. First we deal with the nonrelativistic case where $p^{2} / 2 m=E$, here $E$ is the energy and $m$ the mass of an electron. The length of a wave packet is $\Delta x=\frac{\hbar}{\Delta p}=\frac{\hbar p}{6 m \Delta E}$ since $\Delta p=m \Delta E / p$. In the theory of Reference 2 a single electron wave packet splits into three wave packets at the modulating crystal. One packet has the original momentum and energy $p$ and $E$ and the other two have momentum and energy $\dot{p} \pm$, Et where the plus and minus signs indicate a shift up or down in energy respectively. The laser frequency is $\omega$ and the shift in energy has been shown (2) to be such that $E \pm=E \pm \omega$. The distance from the crystal to screen is $D$. Now if two wave packets are to overlap at the target, their relative velocity times transit time must be less than their length. The velocity of the unshifted packet is $p / m$ and the velocities of the shifted packets are $p \pm / m=(p / m)[1 \pm(h \omega / 2 E)]$. The transit time is $0 /(p / m)$. Therefore:

IOne dimension is all that is necessary to make the point presented in this section.

2 Not to be confused with the "Schwarz" inequality. 


$$
(\hbar \omega / 2 E) D<\hbar / \Delta p
$$

or replacing $\Delta p$ by $m \Delta E / p$ and $E$ by $p^{2} / 2 m$

$$
\Delta E<p^{3} / m^{2} \omega D
$$

Another interpretation may be assigned to Equation (i). It was found that the total a.c. current is related to the total d.c. current by Equation (ii)

$$
\vec{J}_{a c}=\vec{J}_{d c} A \cos \left[(\omega / 2 E) \vec{p}^{\prime} \cdot \vec{x}-\ddot{\omega} t+p h a s e\right]
$$

where

$$
\begin{gathered}
A^{2}=\sin ^{2}\left[\frac{1}{8}\left(\frac{\hbar \omega}{{ }^{E}}\right)^{2} \frac{\vec{p}^{\prime} \cdot \vec{x}}{\hbar}\right]\left[\left[\frac{2 e}{m \hbar \omega^{2}} \vec{E}_{0} \cdot\left(\vec{p}-\vec{p}^{\prime}\right)\right]^{2}\right. \\
\left.+\left[\left(4 e E_{O z^{p}} / m \hbar \omega^{2}\right)[(\varepsilon-1) / \varepsilon] \sin (m \omega d / 2 p)\right]^{2}\right\} .
\end{gathered}
$$

Here $\vec{p}^{\prime}$ is the momentum of the scattered electron beam, e the electron charge, $d$ the crystal thickness and $E$ is the magnitude of the electric field in the laser beam. The wave factor in the current is $\cos \left[(\omega / 2 E) \vec{p}^{\prime} \cdot \vec{x}-\omega t\right]$. At a fixed point on the target the phase of the disturbance due to electrons with momentum $p$ is $(\omega / 2 E) p D=m \omega D / p$. The range of phases produced is $m \omega D \Delta p / p^{2}$ and this must be small compared to unity, $m \omega D \Delta p / p^{2}<1$. This again is Equation (i). For $D=30 \mathrm{~cm}$ and using the rest of schiwd $\angle$ 's parameters wo find that $\Lambda F<0.01 \mathrm{eV}$.

By this criterion the electron optics in any experiment done near $50 \mathrm{keV}$ must produce electrons with an energy spread of less than $0.01 \mathrm{eV}$. It is somewhat doubtful that any of the experiments tried thus far 
(including Schwarz's') could have achieved this high degree of resolution. Favro and Kuo (24) made a useful suggestion. Since the resolution needed for a successful experiment is a function of the apparatus energy and since the resolution obtained is determined by use of a thermionic emitter and therefore a constant for all experiments, it might be possible to relax the stringent criterion by going to higher energies.

It is now time to consider what resolution would be needed at higher energy. The calculation must be done relativistically. Here $v=p / \gamma m$ where $\gamma=E / m c^{2}$ and $c^{2} p^{2}=E^{2}-m^{2} c^{4}$. Also if $T$ is the kinetic energy, $E=T+m c^{2}$. Just as before it turns out that $\Delta E<p^{3} / \omega D m^{2}$. But now $P=\left(\frac{T^{2}}{c^{2}}+2 T m\right)^{1 / 2}$. If we consider electrons such that $T=1$ MeV we get $\Delta E<3 \mathrm{eV}$. By going to a higher energy, one that is still available in a few commercial machines, the coherence criterion is relaxed by a factor of 300. Not only that, since the machine would use a thermionic emitter not unlike electron microscopes which operate at a lower energy, the energy uncertainty in the machine should not exceed $1 \mathrm{eV}$ and the ability to modulate the electron beam is an almost certainty. None of the theories espoused thus far have adequately described such modulation at hịgh energy. Van Zandt and Meyer (8) use the Klein-Gordon equation for a preliminary calculation. But at $1 \mathrm{MeV}, v / c>0.94$ for electrons and a relativistic treatment is necessary. Spin must be included in a relativistic treatment and therefore the Dirac equation is the only one appropriate because electrons have spin 1/2. This calculation is carried out in the following chapter.

\footnotetext{
In the author's opinion.
} 


\section{RELATIVISTIC THEORY OF THE SCHWARZ-HORA TYPE MODULATION MECHANISMI, 2}

A. The Laser Potential and General Formulas

It is necessary to write an explicit expression for the four-potential at the crystal where the interactions take place.

$$
A^{\mu}=(U, \AA)
$$

Here $U$ is the lattice scattering operator responsible for the diffraction pattern. The transform of this potential will be used and is defined as

$$
\dot{u}(\vec{q})=\int d^{3} x^{\prime} \dot{u}\left(x^{\prime}\right) e^{i \vec{q} \cdot \vec{x}^{\prime}}
$$

Next consider the laser fields. Suppose the crystal is parallel to the $X Y$ plane, centered at the origin and of thickness $d$. One gets the vector potential from the electric fleld $\vec{E}=-\partial \vec{A} / \partial t, \vec{A}$ is just $-i \vec{E} / \omega$, where $\omega$ is the laser frequency. The crystals used are thin compared to the light wavelength so that reflection effects are small, the laser fields are hardly disturbed by the crystal, and the internal electric field can be found by requiring continuity of the tangential component of the electric field, $\vec{E}_{t a n}$, and the normal component of the displacement, $\vec{b}_{\text {norm }}$. The vector potential may be written as

$$
\vec{A}=\vec{A}_{\ell}+\vec{A}_{n}
$$

'The notation of Bjorken and Drell (32) will be used in this chapter. Also $\hbar=c=1$ unless stated otherwise.

${ }^{2}$ This calculation follows closely in the spirit of the nonrelativistic calculation in Reference 2 . 
where

$$
\vec{A}_{\ell}=\left(\frac{\vec{E}_{0}}{\omega}\right) \cos \omega t
$$

and $\vec{A}_{n}$ is in the $z$ direction with

$$
A_{n 3}=\frac{E_{03}}{\omega} \frac{(1-\varepsilon)}{\varepsilon} \quad r(z) \cos \omega t .
$$

Here $\varepsilon$ is the dielectric constant of the material, $\vec{E}_{0}$ sin $\omega t$ is the field produced by the laser at the crystal, and the function $r(z)$ is unity for $-\frac{1}{2} d<z<\frac{1}{2} d$ and zero otherwise. This breaks the total potential $\vec{A}$ up into a part $\vec{A}_{\ell}$ that gives the contribution $\vec{E}_{0}$ sin wt to the field as if the crystal were not present and into a part $\vec{A}_{n}$ that makes a notch in the $z$ component. The part $\vec{A}_{n}$ affects only $E_{03}$ inside the crystal making it be $\left(E_{03} / \varepsilon\right)$ sin $w t$. The proper place for this potential is found in reviewing some Dirac theory.

The incident electron wave is written as

$$
\phi(x)=(m / E V)^{1 / 2} u(p, s) e^{-i p \cdot x}
$$

where $v$ is the normalization volume, $p^{\mu}$ is $(E, \vec{p})$ where $E=\left(p^{2}+m^{2}\right)^{1 / 2}$, and $s^{\mu}$ is the polarization vector. The Dirac equation applied for the electron field $\psi(x)$ in interaction with the laser field and with the lattice,

$$
(i \not D-m) \psi(x)=e A(x) \psi(x)
$$

The solution in Born approximation to second order is

$$
\psi(x)=\phi(x)+\psi_{\text {sC }}^{(1)}(x)+\psi_{\text {sC }}^{(2)}(x)
$$


where

$$
\begin{gathered}
\psi_{S C}^{(1)}(x)=e \int d^{4} x^{\prime} S_{F}\left(x-x^{\prime}\right) A\left(x^{\prime}\right) \phi\left(x^{\prime}\right), \\
\psi_{S C}^{(2)}(x)=e^{2} \int d^{4} x^{\prime} S_{F}\left(x-x^{\prime}\right) A\left(x^{\prime}\right) \int d^{4} x^{\prime \prime} S_{F}\left(x^{\prime}-x^{\prime \prime}\right) A\left(x^{\prime \prime}\right) \phi\left(x^{\prime \prime}\right),
\end{gathered}
$$

and $S_{F}$ is the Feynman propagator

$$
\begin{aligned}
& S_{F}\left(x-x^{\prime}\right)=-i \theta\left(t-t^{\prime}\right) \int \frac{d^{3} p^{\prime}}{(2 \pi)^{3}} e^{-i p^{\prime} \cdot\left(x-x^{\prime}\right)} \frac{p^{\prime}+m}{2 E^{\prime}} \\
& +i \theta\left(t^{\prime}-t\right) \int \frac{d^{3} p^{\prime}}{(2 \pi)^{3}} e^{i p^{\prime} \cdot\left(x-x^{\prime}\right)} \frac{p^{\prime}-m}{2 E^{\prime}}
\end{aligned}
$$

The second term in the propagator will lead to terms no greater than the first. Only the latter will be considered in this calculation. The function $\theta\left(t-t^{\prime}\right)$ has the value unity for $t^{\prime}<t$ and zero otherwise.

\section{The Undeflected Beam}

The laser modulation of the central electron beam comes from mixing. the zeroth-order function $\phi$ and part of the first-order function $\psi_{s c}^{(1)}$ in Equation (7). The scalar potential $U$ does not contribute to the modulation in first order and only the $\vec{A}_{n}$ term of the vector potential survives the integrations. Then since only the third component of $\vec{A}_{n}$ is nonzero' we combine Equations (5), (8), and (10) to obtain

'The field discontinuity is only in the $z$-direction. 


$$
\begin{gathered}
\phi_{s c}^{(1)}(x)=-e \int d^{4} x^{\prime}(-i) \int \frac{d^{3} p^{\prime}}{(2 \pi)^{3}} e^{-i p^{\prime} \cdot\left(x-x^{\prime}\right)} \frac{p^{\prime}+m}{2 E} \\
r^{3} A_{n 3}\left(x^{\prime}\right)\left(\frac{m}{E V}\right)^{1 / 2} y(p, s) e^{-i p \cdot x^{\prime}} .
\end{gathered}
$$

Now let $A_{n 3}\left(x^{\prime}\right)=A_{n 3}\left(\vec{x}^{\prime}\right) \frac{\omega}{|\omega|} \cos \omega t$,

and define the transform

$$
\vec{A}(\vec{q})=\int d^{3} x^{\prime} e^{i \vec{q} \cdot \vec{x}^{\prime}} \vec{A}\left(\vec{x}^{\prime}\right)
$$

We then have

$$
A_{n 3}(\vec{q})=\frac{2 E_{03}}{\omega} \frac{(1-\varepsilon)}{\varepsilon}(2 \pi)^{2} \delta\left(q_{1}\right) \delta\left(q_{2}\right) \frac{\sin \left[q_{3} \frac{d}{2}\right]}{a_{3}} .
$$

Using Equation (13) in Equation (11) and doing the integrals over dx' we get

$$
\begin{aligned}
& \underset{\substack{\psi(x) \\
\dot{\psi}(x)}}{(1)}=\frac{(-i e)}{2|\omega|} \frac{(1-\varepsilon)}{\varepsilon} E_{03} \int d^{3} p^{\prime} \delta\left(p_{1}-p_{1}{ }^{\prime}\right) \delta\left(p_{2}-p_{2}{ }^{\prime}\right) \\
& \frac{\sin \left[\frac{\left(p_{3}-p_{3}{ }^{\prime}\right) d}{2}\right]}{p_{3}-p_{3}^{\prime}} \delta\left(E^{\prime}-E+\omega\right) \frac{p^{\prime}+m}{E^{\prime}}\left(\frac{m}{E V}\right)^{1 / 2} e^{-i p^{\prime} \cdot x} \\
& \gamma^{3} w(p, s)+\{w+-w\}
\end{aligned}
$$

where the symbol $\{\omega \rightarrow-\omega\}$ means the exact same term as precedes it but with 
$\omega$ replaced by $(-\omega)$.

Some tricks must be played with the delta function $\delta(E:-E+\omega)$ to explicitly express it in terms of $p^{\prime}$ (see Appendix 1). Then

$$
\delta\left(E^{\prime}-E+\omega\right)=\frac{\delta\left(p_{3}^{\prime}-\sqrt{p_{3}^{2}-2 \omega E}\right) \cdot \sqrt{E^{2}-2 \omega E}}{\sqrt{P_{3}^{2}-2 \omega E}}
$$

Performing the integrals $\int d^{3} p^{\prime}$ leaves

$$
\begin{aligned}
& \psi \underset{s c}{(1)}(x)=\frac{-i e}{2} \frac{(\varepsilon-1)}{\varepsilon} \frac{E_{03}}{|\omega|}\left(\frac{m}{E V}\right)^{1 / 2} \cdot \frac{\sin \left(\frac{\omega E}{2 p_{3}} d\right)}{\omega E / p_{\xi}} \\
& {\left[\gamma^{\circ}\left(E^{2}-2 \omega E\right)^{1 / 2}-\gamma^{3}\left(p_{3}{ }^{2}-2 \omega E\right)^{1 / 2}+m\right] e^{-i p^{\prime} \cdot x} \gamma^{3} u(p, s)} \\
& +\{\omega \rightarrow-\omega\}
\end{aligned}
$$

Now $E_{ \pm}=E \pm \omega$

$$
\left.P_{ \pm}=[E \pm \omega)^{2}-m^{2}\right]^{1 / 2}
$$

and since the third component of the three momentum $\vec{p}$ is the only nonzero component we write $p_{3}=p$.

Then 


$$
\begin{aligned}
& \psi_{s c}^{(1)}(x)=\frac{1 e E_{03} d}{4 \omega} \cdot \frac{(1-\varepsilon)}{\varepsilon}\left(\frac{m}{E V}\right)^{1 / 2}\left[\frac{1}{p_{+}} \frac{\sin \frac{1}{2} d\left(p-p_{+}\right)}{\frac{1}{2} d\left(p-p_{+}\right)}\right. \\
& e^{i\left(p_{+} z-E_{+} t\right)}\left(E_{+} \gamma^{0}-p_{+} \gamma^{3}+m\right) \gamma^{3} u\left(p_{+}, s\right)+\frac{1}{p_{-}} \frac{\sin \frac{1}{2} d\left(p-p_{-}\right)}{\frac{1}{2} d\left(p-p_{-}\right)} \\
& e^{i\left(p_{-} z-E_{-} t\right)}\left(E_{-} \gamma^{0}-p_{-} \gamma^{3}+m\right) \gamma^{3} u\left(p_{-}, s\right)
\end{aligned}
$$

where terms of the order $\frac{\omega}{E}$ in the expansion of $\left(E^{2}-2 \omega E\right)^{1 / 2}$ have been dropped. We make an expansion in the $\beta_{ \pm}$formula for small $\left(2 E \omega / p^{2}\right)$, writing

$$
\begin{aligned}
p_{ \pm} & =\left(p^{2} \pm 2 \omega E+\omega^{2}\right)^{1 / 2} \\
& =p\left[1 \pm \frac{2 E \omega}{p^{2}}+\left(\frac{p}{2 E}\right)^{2}\left(\frac{2 E \omega}{p^{2}}\right)^{2}\right]^{1 / 2} \\
& \cong p\left[1 \pm \frac{1}{2}\left(\frac{2 E \omega}{p^{2}}\right)=\frac{1}{8}\left(\frac{m}{E}\right)^{2}\left(\frac{2 E \omega}{p^{2}}\right)^{2}\right] .
\end{aligned}
$$

As appropriate for a relativistic particle $p / 2 E$ is treated as of order unity. The expansion in Equation (18) applies in the case considered, $\left(2 E \omega / p^{2}\right)$ having a value near $10^{-5}$ for a $1 \mathrm{MeV}$ electron beam. This type of expansion gives a convenient arrangement of the results and specializes to the expansion used in the nonrelativistic problem where $E$ goes to $m$. $'$

The expansion is in Equation (14) of Reference (2). The symbol E there denotes the kinetic energy $p^{2 / 2} \mathrm{~m}$ whereas here it means the kinetic plus rest energy, $\left(p^{2}+m^{2}\right)^{1 / 2}$. 
Different orders of the expansion are appropriate at different places in Equation (17). In the exponent all three terms are used, in ( $p-p_{ \pm}$) two terms are used, and otherwise $P_{ \pm}$is replaced just by $p$ to get the main effect. In the exponent $E_{ \pm}$is replaced by $E_{-} w$ and otherwise it is replaced by $E$. The wave function then looks considerably simpler,

$$
\begin{aligned}
& \underset{S C}{(1)}(x)=-i \frac{e E_{O B} d}{2 \omega p} \frac{\varepsilon-1}{\varepsilon}\left(\frac{m}{E V}\right)^{1 / 2} \frac{\sin \left(\frac{E \omega d}{2 p}\right)}{\left(\frac{E \omega d}{2 p}\right)} e^{i(p z-E t)} \\
& \exp \left[-\frac{1}{8} i\left(\frac{2 m \omega}{p^{2}}\right)^{2} p z\right] \cos \omega\left(\frac{z}{p / E}-t\right) \\
& \left(E \gamma^{0}-p \gamma^{3}+m\right) \gamma^{3} \dot{u}(p, s) .
\end{aligned}
$$

The current in the $Z$ direction for the wave function $\psi(x)=\phi(x)+\psi(x)$ is then $j=\bar{\psi} \gamma^{3} \psi$

$$
\begin{aligned}
j & \cong \Phi \gamma^{3} \phi+\bar{\psi}_{s c}^{(1)} \gamma^{3} \phi+\bar{\phi} \gamma_{\psi}^{3}(1) \\
s c & \\
& =\Phi \gamma^{3} \phi+2 \operatorname{Re} \bar{\phi} \gamma^{3} \psi(1)
\end{aligned}
$$

By inserting the energy projection operator $\frac{\left(E \gamma^{0}-\gamma^{3} p+m\right)}{2 m}$ and llie polarization projection operator $\frac{1}{2}\left(1+\gamma_{5}\right.$ \&) after the $\bar{u}$ factors the terms in Equation (19) can be set up as a trace calculation. 


$$
\begin{aligned}
\bar{\phi} \gamma^{3} \phi & =(4 E V)^{-1} \operatorname{Tr}\left[\left(E \gamma^{0}-\gamma^{3} p+m\right)\left(1+\gamma_{5} \delta\right) \gamma^{3}\right] \\
& =(4 E V)^{-1}(4 p) \\
\bar{\phi} \gamma^{3} \psi_{S C}^{(1)} & =-i \frac{e E_{O z} d}{2 \omega p} \frac{\varepsilon-1}{\varepsilon} \frac{\sin \left(\frac{E \omega d}{2 p}\right)}{\left(\frac{E \omega d}{2 p}\right)} \exp \left[-\frac{1}{8} i\left(\frac{2 m \omega}{p^{2}}\right)^{2} p z\right] \\
\cos \omega\left(\frac{z}{p / E}-t\right) \frac{m}{E V} \frac{1}{4 m} & \operatorname{Tr}\left[\left(E \gamma^{0}-p \gamma^{3}+m\right)\left(1+\gamma_{5} \phi\right) \gamma^{3}\left(E \gamma^{0}-p \gamma^{3}+m\right) \gamma^{3}\right] .
\end{aligned}
$$

The final trace is just $8 p^{2}$. The dependences on the polarization vector $s^{\mu}$ drop out. We end up with the d.c. probability current $\frac{p}{E V}$ with a ripple. Equation (19) then gives

$$
\begin{gathered}
j=j_{d c}\left[1-2 \frac{e E_{o \xi^{d}}^{d}}{\omega} \frac{\varepsilon-1}{\varepsilon} \frac{\sin \left(\frac{E \omega d}{2 p}\right)}{\left(\frac{E \omega d}{2 p}\right)} \sin \frac{1}{8}\left(\frac{2 m \omega}{p^{2}}\right)^{2}\right. \\
\left.\cos \omega\left(\frac{z}{p / E}-t\right)\right] .
\end{gathered}
$$

In the nonrelativistic limit we let $E \rightarrow m$. Then we end up with the same result as in Reference (2) if Equation (17) there is multiplied by $\frac{\mathrm{p}}{\mathrm{m}}$ to give the current and by the inverse of the normalization volume. used here. 


\section{The Diffracted Beams}

To find the ripple in the diffracted beams one combines the first order contribution from the lattice scattering operator $U(\vec{x})$ and two second order contributions where the $U \gamma^{\circ}$ and the $-\vec{A} \cdot \vec{\gamma}$ parts of $A$ are each present in Equation (9). The lattice scattered wave function is $\psi$ lat and is found by putting $\gamma^{0} U\left(\vec{x}^{\prime}\right)$ in Equation (8) for $\alpha\left(x^{\prime}\right)$. Then using the transform defined in Equation (1) and performing the spacial and time integrations" we have

$$
\begin{aligned}
& \left.\psi(x)=-i \text { e } \int \frac{d^{3} p^{\prime}}{(2 \pi)^{2}} \delta\left(E^{\prime}-E\right) \frac{p^{\prime}+m}{2 E^{\prime}} \gamma^{0} U(\vec{p}-\vec{p})^{\prime}\right) \sqrt{\frac{m}{E V}} \\
& e^{-i p^{\prime} \cdot x} u(p, s)
\end{aligned}
$$

Then using the fact $d^{3} p^{\prime}=p^{\prime} E^{\prime} d E^{\prime} d \Omega^{\prime}$ and performing the energy integration,

$$
\underset{\text { lat }}{\psi(x)}=\frac{-i e}{(2 \pi)^{2}}\left(\frac{m}{E V}\right)^{1 / 2} \int \frac{p^{\prime}}{2}\left[\left(p^{\prime}+m\right) \gamma^{o} u(p, s)\right] e^{-i p^{\prime} \cdot x} U\left(\vec{p}-\vec{p}^{\prime}\right) d \Omega^{\prime} .
$$

The second order wave functions sought are $\psi_{s C}^{(2) n}$ where $\vec{A}_{n}$ is important and $\psi_{\text {sc }}^{(2)}$ where $\vec{A}_{\ell}$ is important. Putting the potentials in the appropriate places in Equation (9) we get

$$
\begin{aligned}
\underset{s c}{\psi(2) \ell}= & e^{2} \int d^{4} x^{\prime} d^{4} x^{\prime \prime} S_{F}\left(x-x^{\prime}\right)\left\{\gamma^{\circ} U\left(x^{\prime}\right) S_{F}\left(x^{\prime}-x^{\prime \prime}\right)\left(-\gamma^{3} A_{l 3}\left(x^{\prime \prime}\right)\right)\right. \\
& \left.-\gamma^{3} A_{l 3}\left(x^{\prime}\right) S_{F}\left(x^{\prime}-x^{\prime \prime}\right) \gamma^{O} U\left(x^{\prime \prime}\right)\right\} \phi\left(x^{\prime \prime}\right),
\end{aligned}
$$


and

$$
\begin{aligned}
\underset{s c}{\psi(x)^{(2)}=} & e^{2} \int d^{4} x^{\prime} d^{4} x^{\prime \prime} s_{F}\left(x-x^{\prime}\right)\left\{\gamma^{\circ} U\left(x^{\prime}\right) s_{F}\left(x^{\prime}-x^{\prime \prime}\right)\left(-\gamma^{3} A_{n 3}\left(x^{\prime \prime}\right)\right)\right. \\
& \left.-\gamma^{3} A_{n 3}\left(x^{\prime}\right) S_{F}\left(x^{\prime}-x^{\prime \prime}\right) \gamma^{\circ} U\left(x^{\prime \prime}\right)\right\} \phi\left(x^{\prime \prime}\right) .
\end{aligned}
$$

In Equation (25) we put in the explicit form of the propagator from Equation (10) and perform the time integrals to obtain

$$
\begin{aligned}
& \underset{S C}{\underset{S C}{(2) l} l}=\frac{e^{2}}{(2 \pi)^{5}}\left(\underset{E V}{\left(\frac{m}{m}\right.}\right)^{1 / 2} \frac{E_{03}}{21|\omega|} \int d^{3} x^{1} d^{3} x^{\prime \prime} d^{3} p^{\prime} d^{3} p^{\prime \prime} \\
& e^{i \vec{p}^{\prime} \cdot\left(\vec{x}-\vec{x}^{\prime}\right)} e^{-i E^{\prime} t} \frac{p^{\prime}+m}{2 E^{\prime}}\left\{\gamma^{O} U\left(\vec{x}^{\prime}\right) \frac{p^{\prime \prime}+m}{2 E^{\prime \prime}} \gamma^{j} \frac{1}{E^{\prime \prime}-E+w-i \delta}\right. \\
& \left.+\gamma^{j} \frac{p^{\prime \prime}+m}{2 E^{\prime \prime}} \gamma^{0} U\left(\vec{x}^{\prime \prime}\right) \frac{1}{E^{\prime \prime}-E-i \delta}\right\} \delta\left(E^{\prime}-E+\omega\right) \\
& e^{i \vec{p}^{\prime \prime} \cdot\left(\vec{x}^{\prime}-\vec{x}^{\prime \prime}\right)} e^{i \vec{p} \cdot \vec{x}^{\prime \prime}} u(p, s)+\{\omega+-\omega\},
\end{aligned}
$$

and

$$
\begin{aligned}
& \underset{S C}{\underset{S C}{(2) n}}=\frac{e^{2}}{(2 \pi)^{5}}\left(\frac{m}{E V}\right) \frac{1}{2.1} \int d^{3} x^{\prime} d^{3} x^{\prime \prime} d^{3} p^{\prime} d^{3} p^{\prime \prime} e^{i \vec{p}^{\prime} \cdot\left(\vec{x}-\vec{x}^{\prime}\right)} \\
& \frac{p+m}{2 E^{\prime}}\left\{\gamma^{\circ} U\left(x^{\prime}\right) \frac{p^{\prime \prime}+m}{2 E^{\prime \prime}} \frac{\gamma^{3} A_{n 3}\left(\vec{x}^{\prime \prime}\right)}{E^{\prime \prime}-E+\omega-1 \delta}+r^{3} \frac{p^{\prime \prime}+m}{2 E^{\prime \prime}} \gamma^{O} U\left(x^{\prime \prime}\right)\right.
\end{aligned}
$$




$$
\begin{aligned}
& \left.\frac{A_{n 3}\left(\vec{x}^{\prime}\right)}{E^{\prime \prime}-E-i \delta}\right\} \delta\left(E^{\prime}-E+\omega\right) e^{i \vec{p}^{\prime \prime} \cdot\left(\vec{x}^{\prime}-\vec{x}^{\prime \prime}\right)} e^{i \vec{p} \cdot \vec{x}^{\prime \prime}} u(p, s) \\
& +\{\omega \rightarrow-\omega\} .
\end{aligned}
$$

Here $\delta$ is an arbitrarily small number. The spatial integrations are then performed, the integrals over $d^{3} p^{\prime \prime}$ are done in the complex plane such that all appearing exponentials converge, the integrals on $d^{3} p^{\prime}$ are reduced to one over the solid angle $d \Omega^{\prime}$, and approximations are made exactly as in the first order case. ' Equations (26) then become

$$
\begin{aligned}
& \underset{S C}{\underset{S C}{(2) l} l}=\frac{-e^{2}}{(2 \pi)^{2}}\left(\frac{m}{E V}\right)^{1 / 2} \frac{1}{\omega^{2}} \int d \Omega^{\prime} p^{\prime} \frac{p^{\prime}+m}{2} U\left(\vec{p}-\vec{p}^{\prime}\right) \\
& \left\{\gamma^{\circ} \frac{\not+m}{2 E} \gamma^{j} E_{O j}-\gamma^{j} E_{O j} \frac{p^{\prime}+m}{2 E^{\prime}}\right\} e^{i\left(\overrightarrow{p^{\prime}} \cdot \vec{x}-E t\right)} e^{-i \frac{1}{2}} \frac{\omega^{2} m^{2} \vec{p}^{\prime} \cdot \vec{x}}{|p|^{4}} \\
& \sin \left[\frac{\omega E}{|p|^{2}} \vec{p}^{\prime} \cdot \vec{x}-\omega t\right] u(p, s),
\end{aligned}
$$

and

'See paragraph immediately following Equation (18) Section B of this chapter. 


$$
\begin{aligned}
& \underset{S c}{(2) n}=\frac{i \pi e^{2}}{4 \omega^{2}} \frac{1}{(2 \pi)^{3}} \frac{\varepsilon-1}{\varepsilon}\left(\frac{m}{E V}\right)^{1 / 2} \frac{p}{E} \int d \Omega^{\prime} U\left(\vec{p}-\vec{p}^{\prime}\right) \\
& \left\{( p ^ { \prime } + m ) Z _ { 0 } ( p ^ { \prime } + m ) \gamma ^ { 0 } \left[\left(1-e^{\frac{i \omega d E}{2 p}}\right) \exp \left[-i\left(\frac{\omega E}{|p|^{2}} \vec{p}^{\prime} \cdot \vec{x}-\omega t\right)\right]\right.\right. \\
& \left.-\left(1-e^{\frac{-i \omega d E}{2 p}}\right) \exp \left[i\left(\frac{\omega E}{|p|^{2}} p^{\prime} \cdot x-\omega t\right)\right]\right] \\
& +\left(p^{\prime}+m\right) \gamma^{0}(\not p+m) Z_{0}\left[\left(e^{\frac{-i \omega d E}{2 p}}-1\right) \exp \left[-i\left(\frac{\omega E}{|p|^{2}} \vec{p}^{\prime} \cdot \vec{x}-\omega t\right)\right]\right. \\
& \left.-\left(e^{\frac{i \omega d E}{2 p}}-1\right) \exp \left[i\left(\frac{\omega E}{|p|^{2}} \vec{p}^{\prime} \cdot \vec{x}-\omega t\right)\right]\right] e^{i\left(\vec{p}^{\prime} \cdot \vec{x}-E t\right)} \\
& \left.e^{-\frac{i}{8}}\left(\frac{2 \omega m}{|p|^{2}}\right) \vec{p}^{\prime} \cdot \vec{x}\right\} u(p, s) \\
& \vec{p}^{\prime} \text { has magnitude }|\vec{p}| \text { in Equation (27a) but differs in direction } \\
& \text { from } \vec{p} \text {. In both Equations (27) } \vec{p}^{\prime} \text { is the scattered momentum. The } \\
& \text { exponentials in the brackets in Equation (27b) combine to give terms in- } \\
& \text { volving the product } \sin \left(\frac{E \omega d}{2 p}\right) \cos \omega\left(\frac{p^{\prime} \cdot x}{p^{2} / E}-t\right) \text { when the density is } \\
& \text { found. }
\end{aligned}
$$$$
\text { Next the densities are sought by calculating }
$$ 


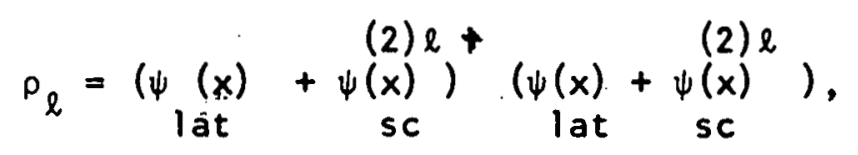

and

$$
\left.\left.\rho_{n}=\underset{\text { lat }}{(\psi(x)}+\underset{\text { sc }}{\psi(2) n}\right)^{\dagger} \underset{\text { lat }}{(\psi}(x)+\underset{\text { sc }}{\psi(x)}\right)
$$

The projection operators for energy and polarization are put in as In the case of the undeflected beam. This reduces the problem to finding some simple traces. Some of these are evaluated in Appendix II. A specific direction is picked to eliminate integrals on solid angle. If the expressions are renormalized such that

$$
\begin{array}{cc}
f^{\dagger}(x) & \psi(x)=1 \\
\text { lat } & \text { lat }
\end{array}
$$

we obtain

$$
\begin{aligned}
& \rho_{\ell}=1-\frac{2 e}{\omega^{2} E} \vec{E} \cdot\left(\vec{p}-\vec{p}^{\prime}\right) \sin \omega\left[\frac{\vec{p}^{\prime} \cdot \vec{x}}{|p|^{2} / E}-t\right] \sin \left[\frac{1}{8}\left(\frac{2 \omega E}{|p|^{2}}\right)^{2} \vec{p}^{\prime} \cdot \vec{x}_{x}\right] \\
& \rho_{n 1}=1-\frac{2 E_{03} d}{\omega} \frac{\varepsilon-1}{\varepsilon} \frac{\sin \frac{E \omega d}{2 p}}{\frac{E \omega d}{2 p}} \sin \left[\frac{1}{8}\left(\frac{2 \omega m}{|p|^{2}}\right)^{2} \vec{p}^{\prime} \cdot \vec{x}\right] \\
& \cos \left[\omega\left(\frac{\overrightarrow{p^{\prime} \cdot \vec{x}}}{|p|^{2} / E}-t\right)\right] .
\end{aligned}
$$

The results may then be easily compared to the nonrelativistic results 
found in Equation (24) and Equation (25) in Reference 2. The only difference is that in the relativistic case an additional factor of $(\mathrm{m} / \mathrm{E})$ is found In all ripple amplitudes. The dependence on crystal thickness is as $\sin \left(\frac{E \omega d}{2 p}\right)$ instead of as $\sin \left(\frac{m \omega d}{2 p}\right)$. The dependence on crystal-to-screen distance $D$ is as $\sin \frac{1}{8}\left(\frac{2 m w}{|p|^{2}}\right)^{2}$ pD in both cases. The ripple moves with the relativistic particle velocity $/ E$.

In specifying the currents found, there is an effect proportional to the component of laser polarization in the direction of the momentum transfer. When it is dominant the current formula is found to be

$$
\begin{gathered}
j=j_{d c}\left[1-\frac{2 e}{E \omega^{2}} \vec{E}_{0} \cdot\left(\vec{p}-\vec{p}^{\prime}\right) \sin \frac{1}{8}\left(\frac{2 m \omega}{|p|^{2}}\right)^{2} \vec{p}^{\prime} \cdot \vec{x}\right. \\
\left.\sin \omega\left(\frac{\vec{p}^{\prime} \cdot \vec{x}}{|p|^{2} / E}-t\right)\right]
\end{gathered}
$$

There is also an effect proportional to the component of laser polarization in the direction of the incident electrons. normal to the crystal. When it dominates the current is

$$
\begin{gathered}
j=j_{d c}\left[1-2 \frac{e E_{03} d}{\omega} \frac{\varepsilon-1}{\varepsilon} \frac{\sin \left(\frac{E \omega d}{2 p}\right)}{\left(\frac{E \omega d}{2 p}\right)} \sin \frac{1}{8}\left(\frac{2 m \omega}{|p|^{2}}\right)^{2} \vec{p}^{\prime} \cdot \vec{x}\right. \\
\left.\cos \omega\left(\frac{\vec{p}^{1 \cdot \cdot \vec{x}}}{|p|^{2} / E}-t\right)\right] .
\end{gathered}
$$


It is interesting to consider relativistic electrons at 1 MeV kinetic energy and compare with nonrelativistic electrons at $50 \mathrm{keV}$. At I MeV the ratio $\mathrm{E} / \mathrm{m}$ is three and the momentum is a factor of six larger than at $50 \mathrm{keV}$. Considering that the amplitude is proportional to $\mathrm{p} / \mathrm{E}$, it is a factor of two larger in the relativistic case. Since the wavelength of the crystal-to-screen dependence is proportional to $p^{3}$, it will be 250 times longer. It is easy to assess the value of doing the experiment at $1 \mathrm{MeV}$ over dolng it at $50 \mathrm{keV}$. The coherence criterion is relaxed so that existing electron optics can produce an adequate electron beam. The amplitude is enhanced by a factor of two. And with the new crystal-toscreen dependence it is not likely that the detector would be sitting at a null while the apparatus is being tuned up. 
VII. FINAL REMARKS

Scientific detectives are still investigating the case of the missing blue dots. Clues provided by experimental and theoretical specialists indicate that the original report was a false alarm. However, the only witness for the defense still claims to have seen spots before his eyes. Lack of evidence will probably keep the case from ever being closed. Each reader of this manuscript is a juror. Regardless of the verdict it is obvious that much has been learned and perhaps a new branch of technology has been formed with the indication that the use of higher energy may enlighten the future. 


\section{REFERENCES CITED}

1. R. Hadley, D. W. Lynch, E. Stanek, and E. A. Rosauer, Appl. Phys. Letters 19, 145 (1971).

2. G. R. Hadley, E. J. Stanek, and R. H. Good, Jr., J. Appl. Phys. 43, 144 (1972).

3. G. R. Hadley, E. J. Stanek, and R. H. Good, Jr., "Discussion of the Schwarz-Hora Effect 11. Relativistic Treatment," lowa State University preprint.

4. H. Schwarz and H. Hora, Appl. Phys. Letters 15, 349 (1969).

5. H. Schwarz, Trans. N.Y. Acad. Sci. 31, 150. (1971).

6. P. L. Rubin, JETP Letters 11, 239 (1970).

7. M. B. 01 iver and L. S. Cutler, Phys. Rev. Letters 25, 273 (1970).

8. L. L. Van Zandt and J. W. Meyer, J. Appl. Phys. 41, 4470 (1970).

9. A. R. Hutson, Appl. Phys. Letters 17, 343 (1970).

10. A. Salat, J. Phys. C3, 2509 (1970).

11. A. D. Varshalovich and M. 1. D'yakanov, Sov. Phys. JGTP Letters II, 594 (1970).

12. L. L. Van Zandt, Appl. Phys. Letters 17, 345 (1970).

13. H. Hora and H. Busch, article to be published in "Advances in Electronics and Electron Physics," (Academic Press, London. Vol. 30A, 1972).

14. H. Schwarz (paper given at the Cleveland APS Meeting, April, 1971).

15. M. H. Pirenne, in The Eye, edited by H. Darson (Academic, New York, 1962), Vol. 2, p. 3, especlally p. 94.

16. Physics Today 24, 17 (June, 1971).

17. H. Schwarz, App1. Phys. Letters 19, 148 (1971).

18. L. Pfeiffer, D. L. Rousseau, and A. R. Hutson, Appl. Phys. Letters 20, $147(1972)$.

19. H. Schwarz, Appl. Phys. Letters, 20, 148 (1972). 
20. L. D. Favro, D. M. FradkIn, and P. K. Kuo, Phys. Rev. Letters 25, $202(1970)$.

21. L. D. Favro, D. M. Fradkin, and P. K. Kuo, Phys. Rev. 3, 2934 (1971).

22. L. D. Favro, D. M. Fradkin, P, K. Kuo, and W. B. Rolnick, Appl. Phys. Letters $18,352(1971)$.

23. L. D. Favro, D. M. Fradkin, P. K. Kuo, and. W: B. Rolnick, Appl. Phys. Letters 19, 378 (1971).

24. L. D. Favro and P. K. Kuo, Phys. Rev. 3, 2931 (1971).

25. C. Becchi and G. Morpurgo, Phys. Rev. D4, 288 (1971).

26. D. Marcuse, J. Appl. Phys. 42, 2255 (1971).

27. D. Marcuse, J. Appl. Phys. 42, 2259 (1971).

28. J. Kondo, J. Appl. Phys. 42, 4458 (1971).

29. H. J. Lipkin and M. Peshkin, Appl. Phys. Letters 19, 313 (1971).

30. G. R. Hadley, "A Theoretical Treatment of the Schwarz-Hora Effect," Ph.D. thesis, lowa State University (1972) (unpublished).

31. R. W. Schmieder, "Interference Effects in Laser Modulated Electron Beams," University of Cal ifornia, Berkeley, preprint.

32. J. D. Bjorker and S. D. Drell, "Relativistic Quantum Mechanics," (McGraw-Hill, New York, 1964). 


\section{ACKNOWLEDGMENT}

The author wishes to express his deeply felt gratitute to Professor R. H. Good, Jr. for his suggestion of and guidance on this and other problems. His generosity in providing insights and encouragement throughout the author's career as a graduate student can never be forgotten. 


\section{APPENDIX I}

$$
\theta\left(E^{\prime}-E+\omega\right)=B\left[\left(p_{3}^{12}+m^{2}\right)^{1 / 2}-\left(p_{3}^{2}+m^{2}\right)^{1 / 2}+\omega\right]
$$

Use the fact that

$$
\theta(g(x))=\sum_{i} \frac{B\left(x-x_{i}\right)}{\left|g^{\prime}\left(x_{i}\right)\right|} \text { where the } x_{i} \text { are zeroes of the function } g(x) \text {. }
$$

$g$ ' is the first derivative of $g$ with respect to its argument. Here

$$
\begin{aligned}
& g\left(p_{3}^{\prime}\right)=\left(p_{3}^{\prime 2}+m^{2}\right)^{1 / 2}-\left(p_{3}^{2}+m^{2}\right)^{1 / 2}+w \\
& g^{\prime}\left(p_{3}^{\prime}\right)=\left(p_{3}^{\prime}\right)\left(p_{3}^{\prime 2}+m^{2}\right)^{-1 / 2} .
\end{aligned}
$$

The zeroes occur at $p_{3}^{\prime}= \pm\left(p_{3}^{2}-\omega^{2}-2 \omega E\right)^{1 / 2}$.

Then for $\omega^{2} \ll p_{3}^{2}$, $2 \omega E$ we get

$$
\delta\left(E^{\prime}-E+\omega\right)=\frac{\delta\left[p_{3}^{\prime}-\left(p_{3}^{2}-2 \omega E\right)^{1 / 2}\right]\left(E^{2}-2 \omega E\right)^{1 / 2}}{\left(p_{3}^{\prime 2}-2 \omega E\right)^{1 / 2}}
$$

plus a term dropped which does not contribute because it implies a negative energy particle. 
XI. APPEND IX 11

There are four major traces which materialize in the calculation of $\rho_{\ell}$. If we define $E_{q \mu} \equiv 0, E_{o i}$ then $\gamma^{\mu} E_{q \mu}=-\gamma^{i} E_{o i}=E_{0}$. The first of the traces is

$$
\begin{aligned}
& \operatorname{Tr} \gamma^{\circ}\left(\not^{\prime}+m\right) \gamma^{\circ}\left(\not p^{\prime}+m\right) \gamma^{0}(\not p+m) Z_{0}(\not p+m) . \\
& \text { Define } \tilde{p}=p_{0},-\vec{p}
\end{aligned}
$$

The trace is then

$$
\begin{aligned}
& \operatorname{Tr}\left(\not^{\prime}+m\right)\left(\not \not^{\prime}+m\right) \gamma^{O}(\not p+m) \mathbb{R}_{0}(\not p+m) \\
& =\operatorname{Tr}\left[\widetilde{p}_{1} \not p^{\prime} \gamma^{O} \not \mathbb{Z}_{0} \not p+\widetilde{p}^{\prime} \not^{\prime} \gamma^{\circ} \not \mathbb{Z}_{0} m+\widetilde{p}^{\prime} \not^{\prime} \gamma^{\circ} \mathbb{Z}_{0} \not p_{m}\right.
\end{aligned}
$$

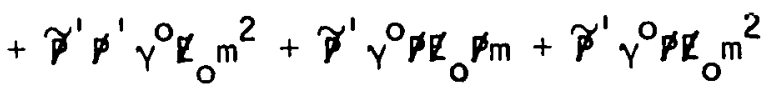

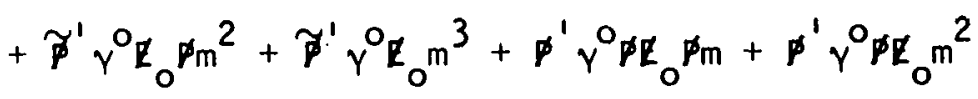

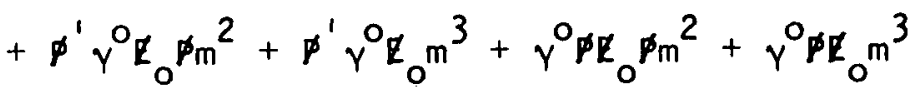

$$
\begin{aligned}
& \left.+\gamma^{O} E_{0} \not m^{3}+\gamma^{O} \not Z_{0} m^{4}\right] \text {. }
\end{aligned}
$$

Consider $\gamma^{0} \equiv \mathbb{Z}$ where $I=1, \overrightarrow{0}$. The trace of a sum is the sum of the traces. The trace of the product of an odd number of $\gamma$ matrices is zero. Therefore only the terms involving even powers of $m$ survive in Equation (A.2). 
Using the general trace theorem

$$
\begin{aligned}
\operatorname{Tr} a_{1} \cdots a_{n} & =a_{1} \cdot a_{2} \operatorname{Tr} b_{3} \cdots a_{n}-a_{1} \cdot a_{3} \operatorname{Tr} \phi_{2} a_{4} \cdots a_{n} \\
& +\cdots+a_{1} \cdot a_{n} \operatorname{Tr} a_{2} \cdots a_{n-1}
\end{aligned}
$$

we find

$$
\begin{aligned}
& \operatorname{Tr}\left[\not^{\prime} \not p^{\prime} \gamma^{\circ} \not E_{0} \not\right]=\not^{\prime} \cdot \not^{\prime} \operatorname{Tr} \gamma^{O} \not E_{0} \not p-\not b^{\prime} \cdot I \operatorname{Tr} \not p^{\prime} \not E_{0} \not p
\end{aligned}
$$

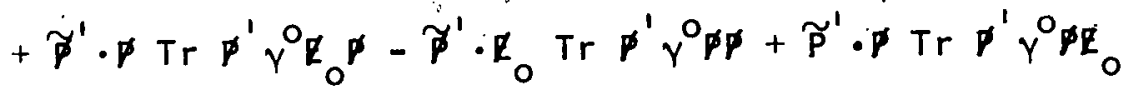

now using Equation (A.3) again

$$
\begin{aligned}
& \operatorname{Tr} \gamma^{\circ} \not \not E_{0} \not=-8 \mathrm{E}_{0} \cdot \overrightarrow{\mathrm{p}} \\
& \operatorname{Tr} \phi^{\prime} \not p E_{0} \phi=4 p \cdot p \vec{E}_{0} \cdot \vec{p}^{\prime}-8 p^{\prime} \cdot p \vec{E}_{0} \cdot \vec{p} \\
& \operatorname{Tr} p^{\prime} \gamma^{\circ} \underline{E}_{0} \not p=-4 E^{\prime} \vec{E}_{0} \cdot \vec{p}-4 E \vec{E}_{0} \cdot \vec{p}^{\prime} \\
& \operatorname{Tr} p^{\prime} \gamma^{O} \not p p=4 E p \cdot p \\
& \operatorname{Tr} \not p^{\prime} \gamma^{\circ} \not E_{0}=-4 E \vec{E}_{0} \cdot \vec{p}-4 \vec{E}_{0} \cdot \vec{p}^{\prime}
\end{aligned}
$$

now putting Equations (A.5-A.9) in Equation (A.4) we have

$$
\begin{aligned}
& \operatorname{Tr}\left[\vec{p}^{\prime} p^{\prime} \gamma^{O} \not E_{0} \not p\right]=-E\left(\overrightarrow{E_{0}} \cdot \vec{p}\right) \quad\left\{8|\vec{p}|^{2}+8 E^{2}+16 \vec{p} \cdot \vec{p}\right\} \\
& +E\left(\vec{E}_{0} \cdot \vec{p}^{\prime}\right)\left\{8|\vec{p}|^{2}-8 E^{2}\right\} \\
& \operatorname{Tr} \widetilde{P}^{\prime} \not^{\prime} y^{\circ} \mathbb{E}_{0} m^{2}=m^{2}\left[8 E \vec{E}_{0} \cdot \vec{p}^{\prime}\right]
\end{aligned}
$$




$$
\begin{aligned}
& \operatorname{Tr} \widetilde{P}^{\prime} \gamma^{O} \not E_{0} m^{2}=4 m^{2}\left[-E \vec{E}_{0} \cdot \vec{p}+E \vec{E}_{0} \cdot \vec{p}^{\prime}\right] \\
& \operatorname{Tr} \widetilde{P}^{\prime} \gamma^{0} \mathbb{R}_{0} P_{m}^{2}=-4 m^{2} E\left[\vec{E}_{0} \cdot \vec{p}+\vec{E}_{0} \cdot \vec{p}^{\prime}\right] \\
& \operatorname{Tr} \not p^{\prime} \gamma^{\circ} \not \mathbb{Z}_{0} m^{2}=-4 m^{2} E\left[\vec{E}_{0} \cdot \vec{p}+\vec{E}_{0} \cdot \vec{p}^{\prime}\right] \\
& \operatorname{Tr} \not p^{\prime} \gamma^{\circ} \underline{z}_{0} \not m^{2}=4 m^{2} E\left[-\vec{E}_{0} \cdot \vec{p}+\vec{E}_{0} \cdot \vec{p}^{\prime}\right] \\
& \operatorname{Tr} \gamma^{O} \not E_{0} \not m^{2}=-8 m^{2} E \vec{E}_{0} \cdot \vec{p} \\
& \operatorname{Tr} \gamma^{\circ} \mathbb{Z}_{0} m^{4}=0
\end{aligned}
$$

Using the right hand sides of Equations (A.10-A.17) in Equation (A.2)

$$
\operatorname{Tr} \gamma^{\circ}\left(\phi^{\prime}+m\right) \gamma^{\circ}\left(\not^{\prime}+m\right) \gamma^{\circ}(\not p+m) \mathbb{E}_{0}(\not p+m)=-16 E\left(\vec{E}_{0} \cdot \vec{p}\right)\left(E^{2}+\vec{p}^{\prime} \cdot \vec{p}+m^{2}\right) .
$$

The next trace to be evaluated is

$$
\operatorname{Tr} \gamma^{O}\left(\not p^{\prime}+m\right) \gamma^{O}\left(\not^{\prime}+m\right) \mathbb{R}_{0}\left(\not \phi^{\prime}+m\right) \gamma^{O}(\not p+m) .
$$

It is similarly evaluated with the result that expression (A.19) equals

$$
-E\left(\vec{E}_{0} \cdot \vec{p}^{\prime}\right) 16\left(E^{2}+\vec{p} \cdot \vec{p}^{\prime}+m^{2}\right)
$$

The next trace is

$$
\operatorname{Tr} \mathbb{R}_{0}(\not p+m) \gamma^{0}\left(\not^{\prime}+m\right) \gamma^{0}\left(\not^{\prime}+m\right) \gamma^{0}(\not p+m)
$$


which is identical to the trace in Equation (A.1) where the cyclic permutation property of traces has been used.

The last trace is

$$
\operatorname{Tr} \gamma^{0}\left(\not p^{\prime}+m\right) \mathbb{E}_{0}\left(\not p^{\prime}+m\right) \gamma^{O}\left(\not p^{\prime}+m\right) \gamma^{O}(\not p+m) .
$$

It is the same as the one in Equation (A.19) with $\widetilde{\beta}^{\prime}$ replaced by $\mathcal{P}$ and: replaced by p'.

Four more traces materialize in the calculation of $\rho_{n}$. The first is

$$
\operatorname{Tr} \gamma^{\circ}\left(\not^{\prime}+m\right) \mathbb{Z}_{0}\left(\not^{\prime}+m\right) \gamma^{\circ}\left(\not^{\prime}+m\right) \gamma^{\circ}(\not{P}+m) .
$$

It is identical to Equation (A. 19) with $\not^{\prime}$ replaced by $\not^{\prime}$ and $\not$ replaced by. .尹.

Then the following trace comes up

$$
\operatorname{Tr} \gamma^{\circ} \mathscr{L}_{0}(\not \not p+m) \gamma^{0}\left(\not^{\prime}+m\right)\left(\not^{\prime}+m\right) \gamma^{\circ}(\not p+m)
$$

along with

$$
\operatorname{Tr} \gamma^{\circ}\left(\not^{\prime}+m\right) \gamma^{O}\left(\not p^{\prime}+m\right) \gamma^{O}(\not p+m) \mathbb{Z}_{0}(\not p+m) \text {. }
$$

Equation (A.24) is exactly the same as Equation (A.25) with replaced by $\widetilde{P}$ and $\not^{\prime}$ replaced by $\widetilde{p}^{\prime}$. Then Equation (A.25) is just exactly the saine as Equation (A.21).

The last trace for $p_{n}$ is

$$
\operatorname{Tr} \gamma^{\circ}\left(\not p^{\prime}+m\right) \gamma^{\circ}\left(\not^{\prime}+m\right) Z_{0}\left(\not^{\prime}+m\right) \gamma^{\circ}(\not p+m)
$$

which is identical to Equation (A.1). 